\title{
Mechanistic diversification of XIST regulatory network in mammals
}

Olga Rosspopoff ${ }^{1}$, Christophe Huret ${ }^{1}$, Amanda J. Collier ${ }^{2,3}$, Miguel Casanova ${ }^{1}$, Peter J. RuggGunn $^{2,3}$, Jean-François Ouimette ${ }^{1 *}$ and Claire Rougeulle ${ }^{1 *}$.

5

${ }^{1}$ Université de Paris, Epigenetics and Cell Fate, CNRS, F-75013 Paris.

2 Epigenetics Programme, The Babraham Institute, Cambridge CB22 3AT, UK.

${ }^{3}$ Wellcome Trust - Medical Research Council Cambridge Stem Cell Institute, University of Cambridge, Cambridge CB2 1QR, UK.

10 *claire.rougeulle@u-paris.fr; jean-francois.ouimette@u-paris.fr

\section{SUMMARY}

$\mathrm{X}$ chromosome inactivation (XCI) is a developmental regulatory process that initiates with remarkable diversity in various mammalian species. Here we addressed the contribution of XCI regulators, most of which are lncRNA genes characterized in the mouse, to this mechanistic diversity. By combining analysis of single-cell RNA-seq data from early human embryogenesis with various functional assays in naïve and primed pluripotent stem cells and in differentiated cells, we demonstrate that $J P X$ is a major regulator of XIST expression in human and in mouse. However, the underlying mechanisms differ radically between species and require Jpx RNA in the mouse and the act of transcription of $J P X$ locus in the human. Moreover, biogenesis of XIST is affected at different regulatory steps between these species. This study illustrates how diversification of LRGs modes of action during evolution provide opportunities for innovations within constrained gene regulatory networks.

\section{KEYWORDS}

$\mathrm{X}$ chromosome inactivation, $X I S T, J P X$, lncRNA, pluripotency, single-cell RNA-seq, human embryogenesis, evolution, gene regulatory networks 


\section{Graphical abstract}

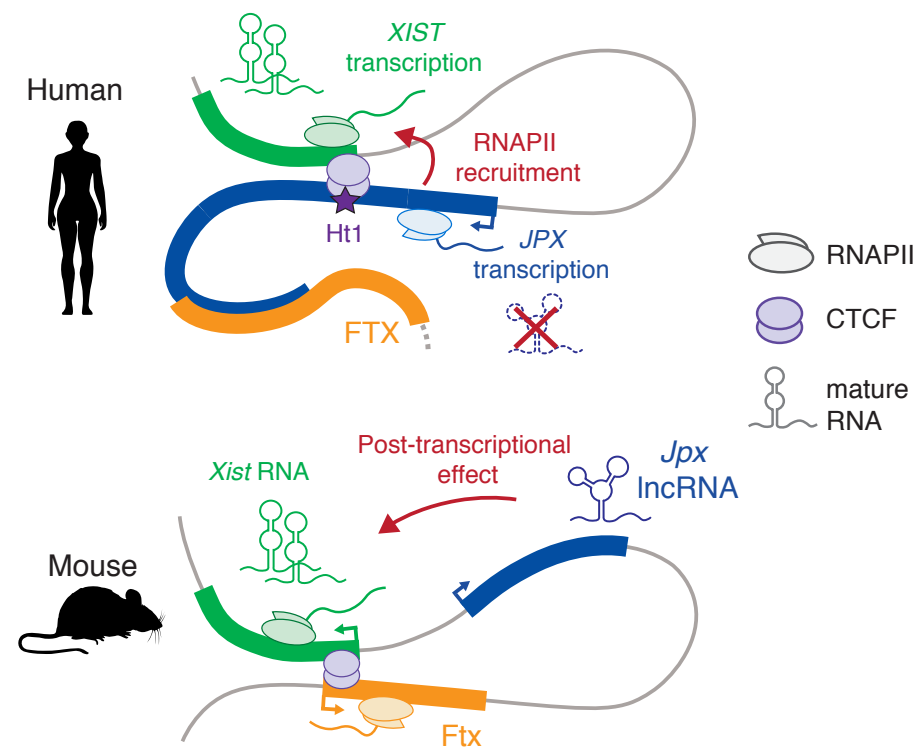




\section{Introduction}

$\mathrm{X}$ chromosome inactivation $(\mathrm{XCI})$ is a fundamental epigenetic process that ensures dosage compensation for $\mathrm{X}$-linked genes expression between male and female mammals. $\mathrm{X}$ chromosome silencing is triggered early in development by the accumulation of the long non-coding RNA (lncRNA) XIST, which acts as a scaffold for multiple protein complexes, involved amongst others in chromatin remodeling, nuclear organization and RNA modification (Furlan and Rougeulle, 2016). The concerted action of these ribonucleoprotein factors results in the conversion of one of the two X chromosomes in females into a compact and transcriptionally silent structure. XIST expression has to be tightly controlled in order to ensure female-restricted inactivation of a single $\mathrm{X}$ chromosome in a timely manner. However, it remains intriguing that such an essential process follows species-specific routes in which the dynamics of XIST expression in early developmental stages differs markedly between mouse and human. While in the mouse Xist is restricted to females and to a single X, XIST expression in human pre-implantation development transiently initiates in a manner that is independent of the sex and of the number of X-chromosomes. XIST accumulation precedes the establishment of proper XCI in human pre-implantation embryos resulting in active XIST-coated Xchromosomes (Okamoto et al., 2011). However, the situation eventually homogenizes and Xist/XIST RNA coating becomes restricted to a single $\mathrm{Xi}$ in both mouse and human post-inactivation (post-XCI) cells (Vallot et al., 2016). These observations raise questions regarding the functional conservation of XIST regulatory network across species. We previously identified the transcription factor YY1 as a 20 potent activator of XIST in mouse and human (Makhlouf et al., 2014), but the activity of the regulatory elements from the genomic region surrounding XIST, known as the X-inactivation center (XIC), has never been addressed in species other than the mouse.

Chromosomal rearrangements in mouse and human have allowed the determination of the physical boundaries of the XIC, defined as necessary and sufficient to trigger XCI. In addition to Xist, other genes were mapped to the mouse Xic, including several protein-coding genes (Slc16a2, Cnbp2, Chic1 and Rnf12) and four additional lncRNA genes (Linx, Tsix, Jpx, and Ftx). While the order and orientation of Xic-linked genes are globally preserved between mouse and human (Chureau et al., 2002; Duret et al., 2006), the human XIC underwent a dramatic expansion and is about three times larger compared to its mouse counterpart. Particularly relevant are the lncRNA genes hosted within the Xic, which have been linked to Xist regulation in the mouse: Tsix and Linx acts as major repressor of Xist expression while Jpx and Ftx acts as positive regulators. The mechanistic dissection of these Xiclinked and other lncRNA loci highlighted that their molecular function is not only mediated by the RNA molecule itself, but may also involve various entities such the act of transcription or key regulatory elements embedded within their locus (Cho et al., 2018; Engreitz et al., 2016; Furlan et 
al., 2018; Paralkar et al., 2016). For instance, the antisense transcription of the Tsix gene over the Xist locus contributes to the monoallelic repression of Xist (Navarro et al., 2005); Tsix transcription is itself controlled by the upstream lncRNA gene, $\operatorname{Linx}$ (Giorgetti et al., 2014; Nora et al., 2012). Ftx transcription has been shown to be essential for Xist expression cis (Furlan et al., 2018) while Jpx was proposed to act through its RNA molecule by binding and titrating away the CTCF protein from the Xist promoter (Sun et al., 2013). Therefore, it appears more appropriate to define loci producing lncRNAs as lncRNA genes (LRGs) to better emphasize their mechanistic versatility. The antagonistic action of the Xic-linked LRGs is likely facilitated by the spatial segregation of the Xist- and Tsixassociated regulators in two adjacent and oppositely regulated topologically associated domains 10 (TADs) (Nora et al., 2012). These TADs also delimit internal long-range interactions to ensure contacts between regulatory elements and their target genes; intra-TAD interactions have been described between the Xist and Ftx LRGs and between Tsix promoter and Linx LRG (Furlan et al., 2018; Nora et al., 2012).

15 The molecular players controlling XCI have been largely characterized in the mouse, in part due to the lack of cellular models recapitulating the early stage of XIST activation in human. While the differentiation of female mouse embryonic stem cells (ESCs) recapitulates Xist upregulation and XCI, no human ex-vivo model faithfully reproduces the biallelic upregulation of XIST observed during in vivo human embryogenesis. Conventional hESCs displays the hallmarks of primed pluripotency including an inactive X-chromosome coated by XIST (Vallot et al., 2016). In addition, loss of XIST expression may occur spontaneously upon prolonged culture of primed hESCs through a process identified as "XCI erosion", that involves the ectopic reactivation of a subset of genes from the Xi (Mekhoubad et al., 2012; Vallot et al., 2015). Nevertheless, the post-XCI context in primed hESCs differ from that of differentiated cells and their use as an XCI model has been proven effective in the identification human-specific XCI regulators such as the lncRNA XACT (Vallot et al., 2013). It is only recently that methods were developed to reset primed hESCs into the naïve state of pluripotency that matches several features of human pre-implantation embryos, including active X-chromosomes coated by XIST (Guo et al., 2017; Sahakyan et al., 2017; Theunissen et al., 2016; Vallot et al., 2016). Indeed, resetting of eroded primed hESCs triggers XIST upregulation concomitantly to $\mathrm{X}$ 30 chromosome reactivation (XCR), although XIST upregulation is often mono-allelic and restricted to the former Xi (Sahakyan et al., 2017; Vallot et al., 2016). While the resetting of these cells is currently the only method to trigger XIST upregulation in human, it has never been used to functionally characterize regulators of XIST induction. Altogether, experimental systems are now available to probe the XIST regulatory network in human. 
From an evolutionary standpoint, one major challenge is that both LRG functionality, if any, and their mechanism of action are hardly predictable based on the DNA sequence alone. The sequence conservation pattern of LRG evolving under functional constraints is therefore difficult to predict. For instance, it is known that syntenic LRG often display strong primary sequence turnover during evolution, even among closely related species (Hezroni et al., 2015; Necsulea et al., 2014; Ulitsky et al., 2011; Washietl et al., 2014), whose impact on LRGs functional conservation is still poorly understood. In rare studies where the functional conservation of lncRNA molecules has been addressed, orthologues display short patches of conserved sequence that are necessary, but not sufficient, for their function (Lin et al., 2014; Ulitsky et al., 2011). While this sharp contrast with the evolutionary stability of protein-coding genes raised controversies on LRGs functionality, experimental investigations have been too limited to provide a definitive understanding of the rules underlying LRGs functional conservation. X-chromosome inactivation provides an interesting experimental paradigm to test this since LRG orthologs are found in the human XIC (Romito and Rougeulle, 2011).

15

In this study, we investigated the regulatory network involved in the initial steps of XIST expression in human during early embryogenesis. The analysis of single-cell RNA-seq data from early human embryos designated $J P X$ as a potent candidate for $X I S T$ regulation in human. Using a panel of functional approaches to target various modules of $J P X$ LRG, we could show that, while human $J P X$ transcripts are dispensable for this process, transcription of the JPX locus is essential to sustain XIST transcription in post-XCI cells. This process is fostered within a sub-TAD domain that involves RNA polymerase II-mediated 3D interactions. By resetting primed hESCs carrying various deletions of $J P X$ promoter region, we demonstrate that this system is suitable to investigate regulators of XIST upregulation in human and identify the JPX LRG as a major cis-regulator of XIST transcriptional activation. We also re-addressed the role of Jpx RNA, matching cellular models and functional approaches between human and mouse. We could identify that Jpx RNA acts as a positive regulator of Xist in mouse post-XCI cells and our findings suggest that Jpx regulates Xist accumulation in a post-transcriptional manner. In addition to identifying of a novel regulator of XIST expression in human, these findings provide a striking demonstration of the mechanistic diversification of orthologous LRGs, which sheds new light on the importance of these noncoding elements in defining species-specific regulatory mechanisms within constrained gene regulatory networks. 


\section{RESULTS}

\section{Identification of candidate regulators of XIST during early human embryonic development.}

To examine whether human XIC-linked genes are involved in the initial upregulation of XIST in vivo, we investigated their expression kinetics during early embryogenesis (Figure 1A), using single-cell

5 RNA-seq datasets obtained from human pre-implantation embryos (Petropoulos et al., 2016; Yan et al., 2013). XIST expression initiates between the four- and eight-cell stages (Figure S1A), corresponding to embryonic day 4 (E4, Figure 1B), and increases hereafter, more predominantly in females than in males. While most of XIC-linked genes remained lowly expressed throughout preimplantation development, $R L I M$ and $J P X$ show the highest levels at the early embryonic days, although they display different expression trajectories (Figure 1C). The expression of the proteincoding gene RLIM is the highest at E3 in both male and female embryos and rapidly decrease in the following days, prior to XIST induction (Figure S1B). This pattern likely reflects strong maternal inheritance of RLIM transcripts, which is consistent with previous observations made in the mouse (Shin et al., 2010). In contrast, low levels of JPX could be detected at the 2-4 cells stage, followed by a major burst of expression at the 8-cell stage (Figure S1A) or E4, coinciding with XIST initial induction (Figure 1D). Except at the E3 stage, JPX was broadly expressed, independently from the sex of the embryos, although $J P X$ levels were almost twice in females compared to male embryos, suggesting an early transcription from the two active X-chromosomes (Figure 1D). Using an RPKM threshold to define XIST and JPX expressing cells in female embryos (Figure 1E), we found that the majority of the cells were expressing either $J P X$ or $J P X$ and XIST concomitantly, with very few XISTonly expressing cells at the early embryonic days (E3 and E4, Figure 1F). This pattern suggests that $J P X$ activation may shortly precede XIST induction and that the two genes become eventually coexpressed in a vast proportion of cells as development progresses. JPX and XIST expression levels were weakly correlated at early stages of embryogenesis (Figure 1G) and within embryonic lineages (Figure $1 \mathrm{H}$ ), indicating that $J P X$ transcriptional activation, but not the level of its RNA products, may be a prerequisite for its function. These results point toward the JPX LRG as a candidate for the regulation of the initial induction of XIST expression during pre-implantation development.

\section{JPX RNA is dispensable for XIST expression in human}

30 The JPX LRG derived from the pseudogenization of the protein-coding gene USPL after the divergence of eutherians and marsupials, and evolved concomitantly to XIST (Elisaphenko et al., 2008; Hezroni et al., 2017), although the two genes display distinct evolutionary trajectories (Figure 2A). While XIST present strong signs of positive selection in both intronic and exonic regions, the $J P X$ LRG evolved through a quasi-neutral selection, as illustrated by a conservation score close to zero along the entire locus (Figure 2B). This strong sequence turnover is essentially due to species- 
A
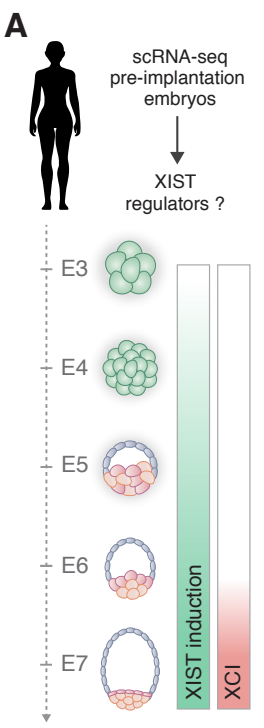

$\mathbf{F}$

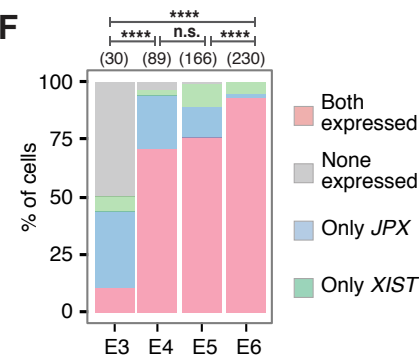

B

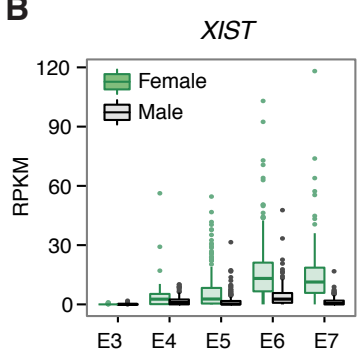

D

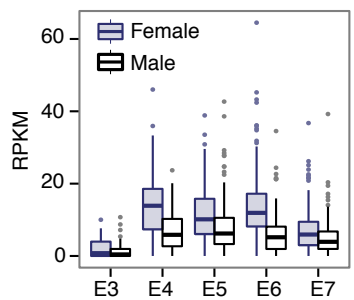

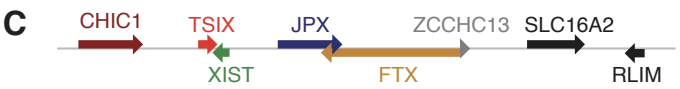

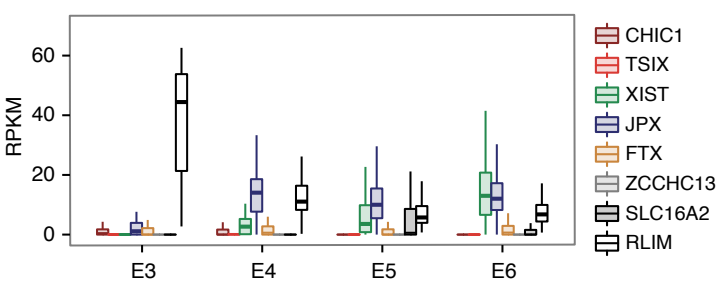

E $\quad \log 2(\mathrm{RPKM} J P X)$

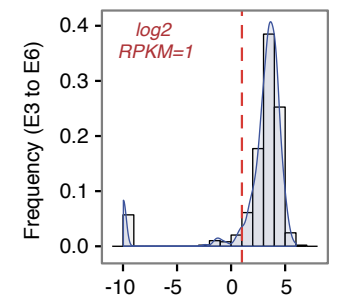

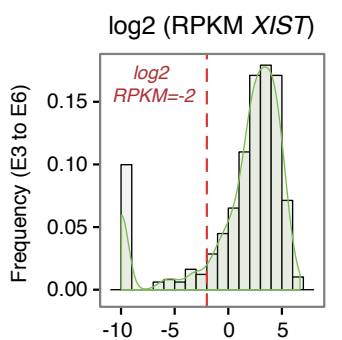
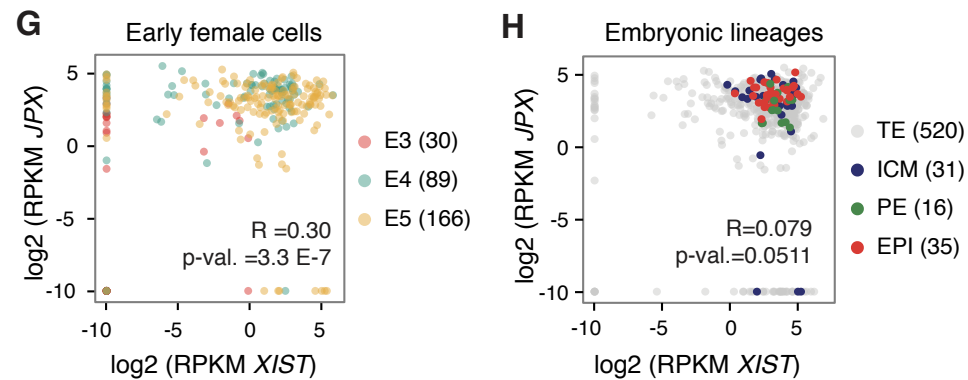

Figure 1. Identification of candidate regulators of XIST during early human embryonic development

(A) Single cell RNA-seq data from E3 to E7 pre-implantation embryos (Petropoulos et al., 2016) were used to probe for XIST regulators. Also shown is the timing of XIST induction along with observed XCI dynamics.

(B) XIST expression is upregulated in male and female embryos from E4. RPKM: Reads Per Kilobase Million

(C) Analysis of single cell expression of $X I C$-linked genes from E3 to E6 reveals that JPX induction precedes that of XIST. (See also Figures S1A and S1B).

(D) $J P X$ is expressed with comparable kinetics in male and female embryos.

(E) The plots represent the distribution of the $\log 2$ RPKM values for JPX and XIST, in female cells from E3 to E6 stages. Red dashed line represents the cutoff used to define JPX and XIST expressing cells, on which panel $(\mathrm{F})$ is based.

(F) Combined analysis of $J P X$ and $X I S T$ expression in single cells showed that the proportion of cells expressing $J P X$ alone decreased during development, while the percentage of cells co-expressing the two genes increased (Chi-square test). n.s., not significant, $* * * \mathrm{p}<0.001 ; * * * * \mathrm{p}<0.0001$.

(G-H) JPX and XIST expression levels were weakly correlated in early stages of embryogenesis and in the different lineages. 

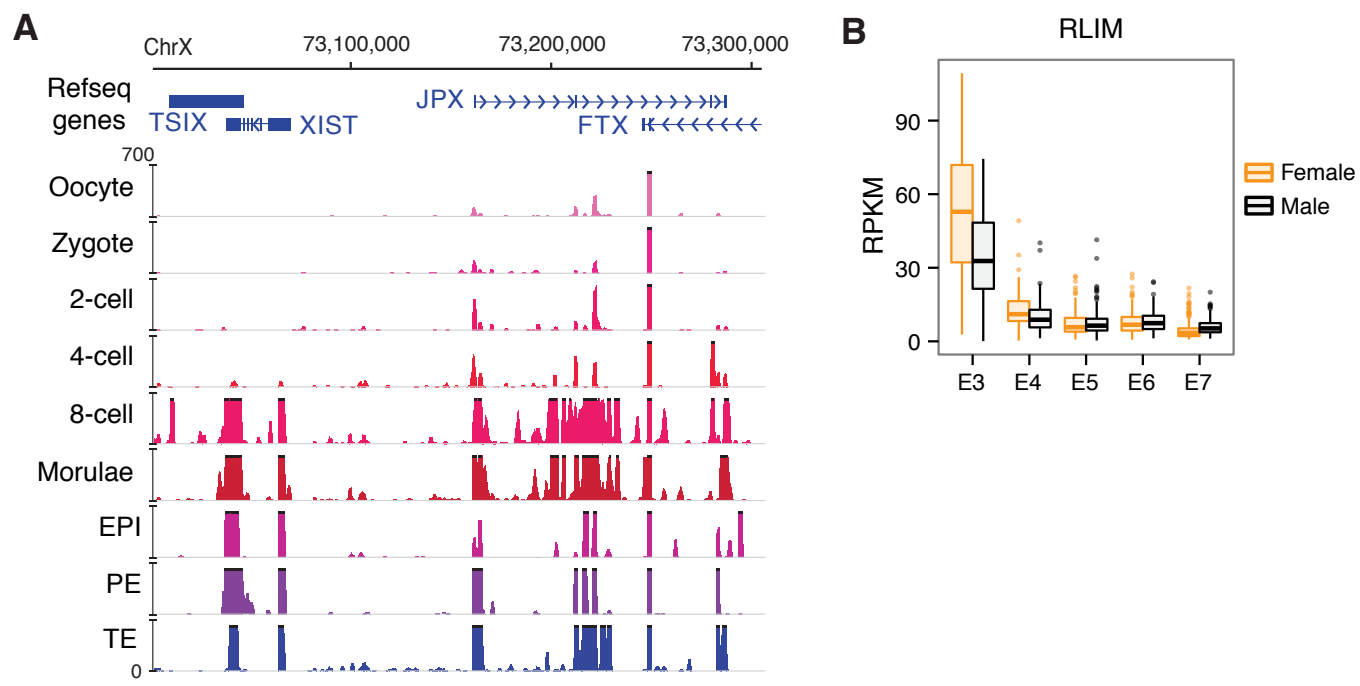

Figure S1: Identification of candidate regulators of XIST during early human embryonic development, related to Figure 1

(A) $J P X$ reads can be detected from the 2-4 cell stages, possibly linked to limited maternal contribution (Yan et al., 2013), but a burst of $J P X$ expression could be seen from the 8-cell stage. At later stages, $J P X$ expression can be detected in the three compartments of the embryos - epiblast (EPI), primitive endoderm (PE) and trophectoderm (TE).

(B) Expression of the protein-coding gene RLIM is the highest at E3 in both male and female embryos and decreases afterwards, suggesting maternal contribution. 
specific integration of transposable elements in this region (Chureau et al., 2002; Kolesnikov, 2010), resulting in poor multiple alignment of the homologous region of five eutherian species (Figure 2B). As observed for numerous LRGs (Hezroni et al., 2017; Hezroni et al., 2015; Washietl et al., 2014), signs of purifying selection on the $J P X$ gene are concentrated toward the promoter region, including the first exon that contains two highly conserved region of $\sim 20$ nucleotides embedded within the mouse and human transcripts. As the human and mouse genes bear limited sequence identity (Chureau et al., 2002), we examined several features of $J P X$ in human, such as its expression pattern and inactivation status in multiple human cell lines. We found that $J P X$ expression is not restricted to pre-implantation development but is ubiquitous across a wide range of human tissues (Figure S2A).

10 Similarly to the pre-XCI state, JPX transcripts levels appeared consistently higher in females compared to males, suggesting expression from both active and inactive $\mathrm{X}$ and, thus, escaping from XCI. To confirm this hypothesis, we performed RNA fluorescence in situ hybridization (FISH) to detect simultaneously sites of $J P X$ active transcription and of XIST RNA accumulation. In both pluripotent and differentiated cellular contexts, $J P X$ was expressed in every cell and a pinpoint of 15 transcription was associated in cis to XIST RNA cloud in about $\sim 80$ to $85 \%$ of cells (Figure $2 \mathrm{C}$ and $\mathrm{S} 2 \mathrm{~B})$, confirming a strong tendency for $J P X$ to escape XCI.

We next analyzed the function of $J P X$ transcripts as they were described as the major functional component in the mouse (Tian et al., 2010). We used an LNA-GapmeR (LGs)-mediated knockdown

20 (KD) strategy (Figure 2D) that have been previously used for the functional analysis of several lncRNAs (Furlan et al., 2018; Leucci et al., 2016; Luo et al., 2016; Tripathi et al., 2010). To address $J P X$ function in an embryonic context, we carried out this analysis in non-eroded primed female hESCs, with a percentage of XIST-expressing cells above 90\% (Vallot et al., 2015). Robust depletion of $J P X$ mature transcripts could be achieved in both H9 and WIBR2 lines (Figure 2E and S2C) using 25 three distinct hLGs targeting $J P X$ second exon, which is common to all of $J P X$ RNA isoforms. As previous studies reported that oligonucleotides containing LNA bases could result in transcriptional inhibition (Beane et al., 2007), we verified that hLGs were not affecting JPX nascent transcription by performing JPX RNA-FISH (Figure 2F), allowing us to address unambiguously the function of the mature transcripts. In both hESCs lines, XIST RNA levels and accumulation within the nuclei 30 remained unaffected by $J P X \mathrm{KD}$, as monitored by RT-qPCR (Figure 2G and S2C) and RNA-FISH (Figure 2H-I and S2D). Similar results were obtained in differentiated cells such as fetal fibroblasts (Figure S2E-F), indicating that JPX mature RNA is dispensable for XIST expression once XCI is established. 
A

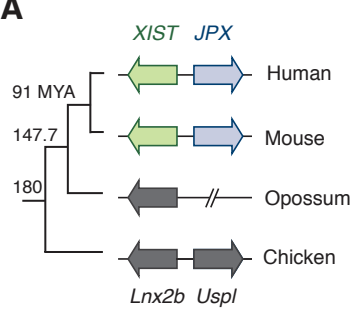

B
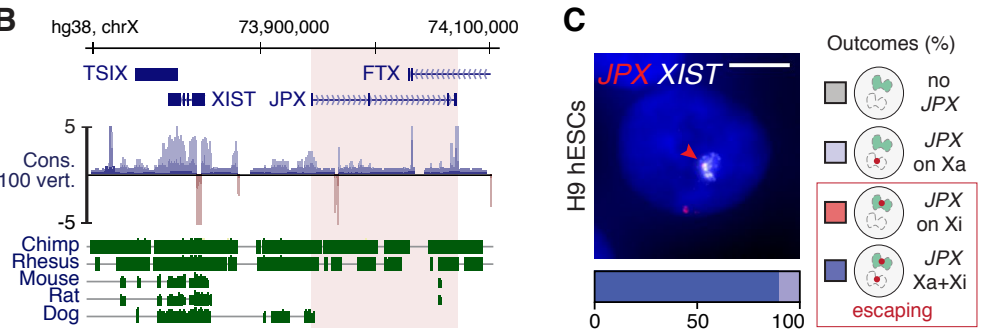

D
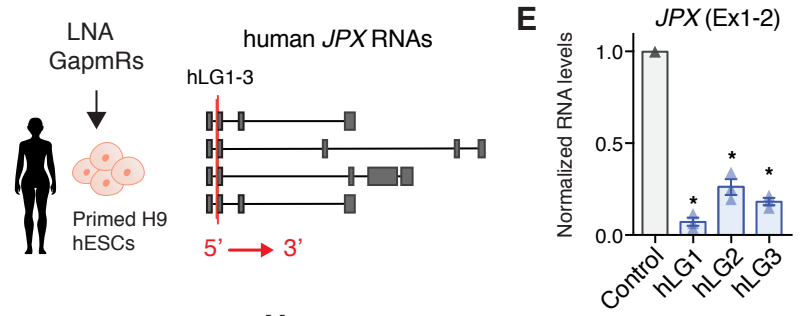

$\mathbf{F}$

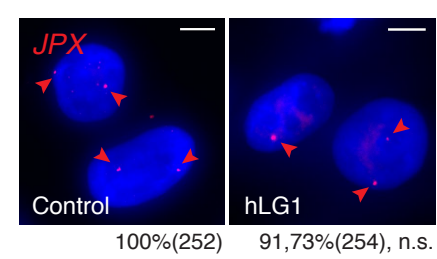

G

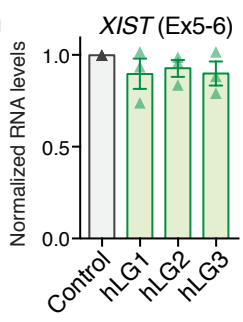

$\mathrm{H}$

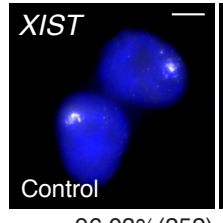

$J$

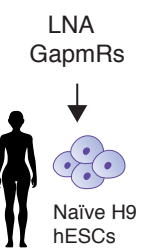

$96,03 \%(252)$

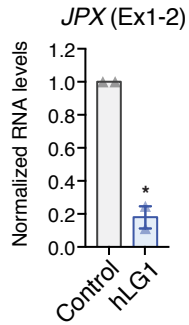

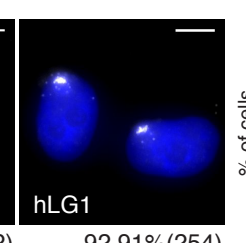
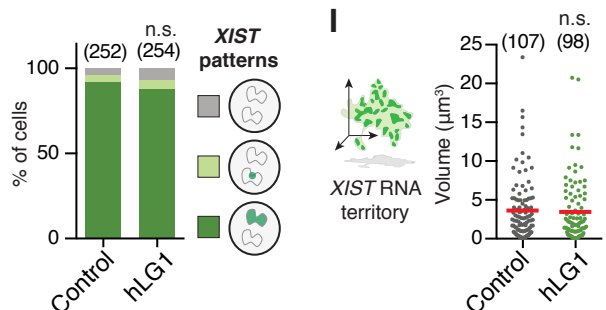

K

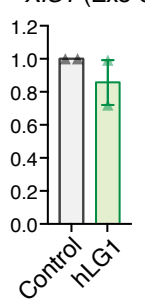

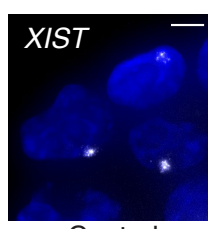

Control

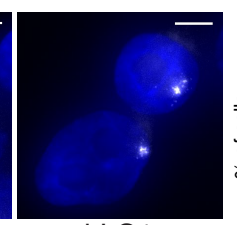

hLG1

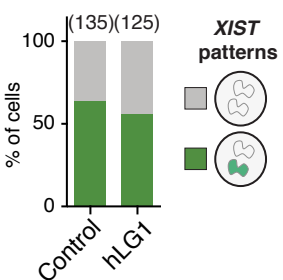

\section{Figure 2: $J P X$ RNA is dispensable for $X I S T$ expression in human}

(A) Scheme of $J P X$ evolution in vertebrates. Colored arrows represent LRGs and grey arrows their proteincoding ancestors.

(B) $J P X$ genomic sequence is weakly conserved, as illustrated by the conservation score in 100 vertebrates and alignment of the human genomic region to five other mammalian species.

(C) $J P X$ escapes $\mathrm{XCI}$ in primed $\mathrm{H} 9 \mathrm{hESCs}$, as assessed by $J P X / X I S T$ double RNA-FISH (see also Figure S2A and S2B).

(D) Scheme of LGs lipofection strategy in primed H9 hESCs and of JPX RNA isoforms (red lines: LGstargeted exons).

(E) $J P X$ RNA levels are reduced following LG transfection, RT-qPCR, $\mathrm{n}=3$ (see also Figure $\mathrm{S} 2 \mathrm{C}$ ).

(F) LGs targeting human $J P X$ RNA did not impact on the number of cells transcribing $J P X$ in primed $\mathrm{H} 9$ hESCs, RNA-FISH. Percentages indicate $J P X$ positive cells, Fischer's exact test.

(G) XIST RNA levels are unaffected following JPX LG-depletion in primed H9 and WIBR2 hESC (see Figure S2C), RT-qPCR, $\mathrm{n}=3$.

(H-I) JPX KD did not affect the number of cells expressing XIST (Chi-square test) or the volume of XIST RNA territory, RNA-FISH, Mann-Whitney test (see also Figure S2D-S2F).

(J) In naïve hESCs, $J P X$ was efficiently downregulated $48 \mathrm{~h}$ post-lipofection (unpaired one-tailed t-test, $\mathrm{n}=2$ ). $J P X \mathrm{KD}$ did not result in changes in XIST RNA levels, RT-qPCR. Expression of pluripotency markers was not affected in these conditions (see Figure S2G), neither was the activity status of the X chromosomes (see Figure S2H).

(K) $J P X$ KD did not impact on the number of cells expressing XIST, RNA-FISH.

Scale bars are $5 \mu \mathrm{m}$. Error bars represent standard deviation; n.s., not significant; ${ }^{*} \mathrm{p}<0.05,{ }^{*} \mathrm{p}<0.01$ and $* * * p<0.001$. Number of counted cells is in brackets. 
A

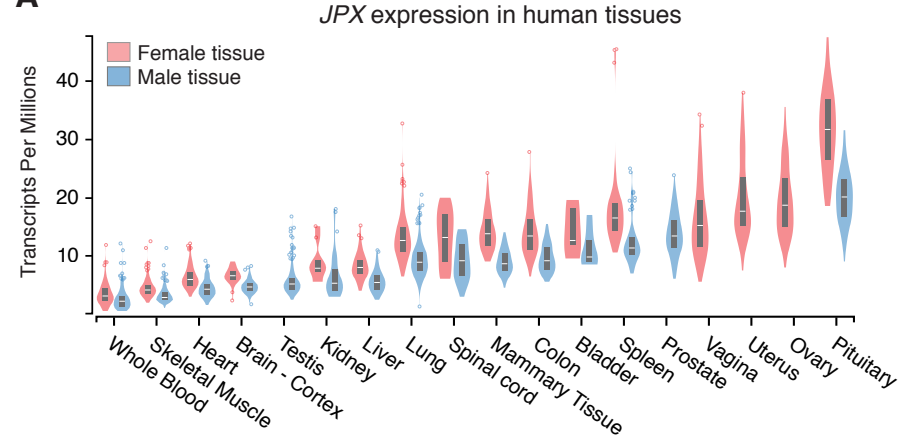

B

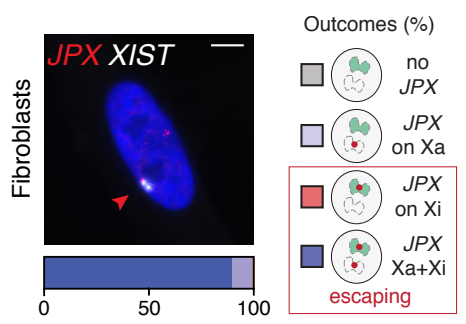

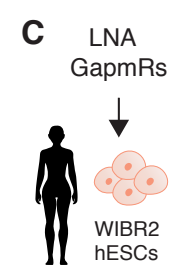
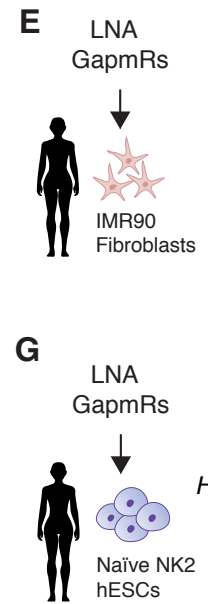
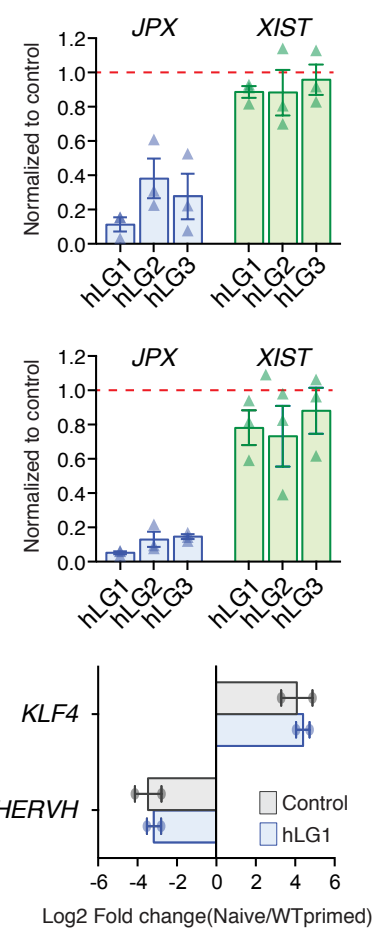

H
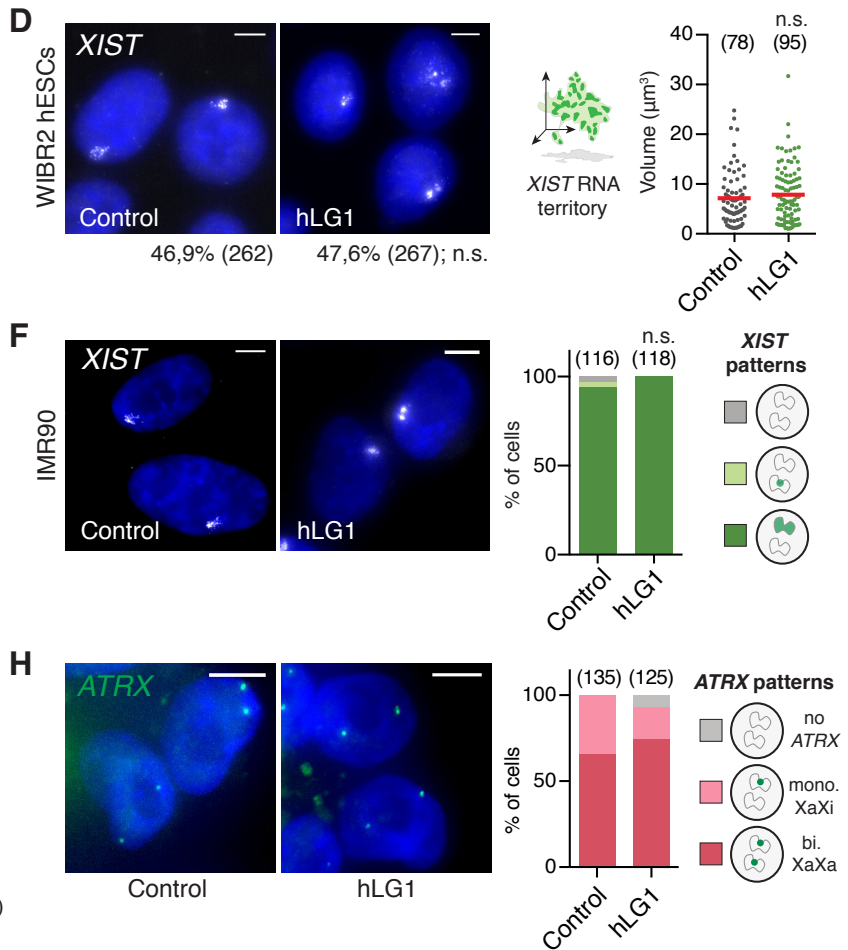

Figure S2: JPX RNA is dispensable for XIST expression in human, related to Figure 2

(A) $J P X$ is ubiquitously expressed across human tissues (Transcripts per Million from the GTEx Project), with $\sim 2$-fold higher expression in female tissues compared to male, in agreement with $J P X$ escaping XCI; the box plots shown as median and 25th and 75 th percentiles over the violon plots.

(B) $J P X$ escapes XCI in female fetal fibroblasts, as assessed by $J P X / X I S T$ double RNA-FISH.

(C-D) KD of human $J P X$ RNA did not impact XIST expression and accumulation in primed WIBR2 hESCs as assessed by RT-qPCR ( $\mathrm{n}=3$ ) and RNA-FISH (Chi square test).

(E-F) XIST RNA levels were not affected by JPX KD in female fetal fibroblasts as assessed by RT-qPCR $(\mathrm{n}=3)$ and RNA-FISH (Chi square test).

(G) $J P X \mathrm{KD}$ did not affect the pluripotency status of the cells as assessed by the quantification of naïve (KLF4) and primed (HERVH) markers, RT-qPCR, $\mathrm{n}=3$.

(H) $J P X \mathrm{KD}$ did not affect X-chromosome activity, as determined by ATRX RNA-FISH (active X marker).

Scale bars are $5 \mu \mathrm{m}$. Error bars represent standard deviation; n.s., not significant. Number of counted cells is in brackets. 
Considering that $J P X$ is broadly expressed during human pre-implantation development, we hypothesized that $J P X$ RNA could function specifically in a pre-XCI context. To test this, we performed $J P X \mathrm{KD}$ in naïve hESCs that were reset from primed hESCs using recently published methods (Sahakyan et al., 2017; Vallot et al., 2017). These cells display XIST-expressing active Xchromosomes and represent the closest in vitro model of pre-XCI as observed in pre-implantation human embryos (Guo et al., 2017; Sahakyan et al., 2017; Theunissen et al., 2016; Vallot et al., 2017). $J P X$ was efficiently knocked-down in naïve hESCs, without impacting on the expression of naïvespecific markers (Figure S2G). In these conditions, neither XIST expression (Figure 2J) nor its pattern of accumulation (Figure 2K) were affected by $J P X$ RNA depletion. We also monitored the activity

10 status of the X-chromosomes by monitoring the ATRX expression by RNA-FISH as its expression only restricted to fully active X-chromosome (Vallot et al., 2015); ATRX transcription remained biallelic in a vast majority of cells suggesting that $J P X$ is not involved in XCI per se (Figure $\mathrm{S} 2 \mathrm{H}$ ). Taken together, these results exclude a function of the JPX RNA in the expression of XIST and XCI in both pre- and post-XCI cells.

15

\section{$X I S T$ expression requires $J P X$ transcription.}

Considering that $J P X$ is the closest gene in 5' to XIST (Johnston et al., 2002), we wondered whether $J P X$ could be part of the XIST cis-regulatory landscape, independently from its RNA transcripts. We characterized long-range interactions surrounding XIST promoter region using published Hi-C (Rao et al., 2014) and ChIA-PET datasets (Ji et al., 2016) generated from female cell lines. This revealed a partitioning of the human XIC into discrete spatial domains that resembled the organization into topological associated domains (TADs) of the mouse syntenic region (Nora et al., 2012) (Figure 3A). Notably, the boundaries of the XIST-associated TAD ( TAD E) fall within XIST locus and upstream of the RLIM gene in both species (Figure 3A). Closer inspection of the XIST-associated TAD in human (Dowen et al., 2014; Hnisz et al., 2016; Ji et al., 2016) revealed that CTCF-CTCF loops formed an insulated chromatin neighborhood hosting preferential contacts between XIST promoter region and the $J P X$ gene (Figure 3B). The CTCF loops anchor a region in the vicinity of XIST TSS (XISTp, $+2,9 \mathrm{~kb})$ to two upstream intragenic regions, or hotspots, located within the $J P X(\mathrm{Ht} 1,+163 \mathrm{~kb})$ and FTX $(\mathrm{Ht} 2,+283 \mathrm{~kb})$ genes. All anchors were co-occupied by CTCF and the cohesin complex, but

30 were not enriched in chromatin marks or proteins associated with active enhancers (Figure S3A). Although a similar organization of the XIST-associated TAD was observed on the sole active $\mathrm{X}$ chromosome of male fibroblasts (Figure S3B), we found that XIST/JPX long-range interactions were associated with RNA polymerase II (RNAPII) only in female datasets (Li et al., 2012), while no peak was reported in that of male (Figure 3B, data not shown). This suggests a female-specific transcriptional association of XIST and $J P X$ that likely occurs on the Xi (Figure 3B). This hypothesis 
is further supported by the high number of cells ( $>80 \%)$ co-transcribing XIST and JPX from the Xi as assessed by RNA-FISH in several cell lines (Figure 2A). These long-range interactions were also detected in CTCF and SMC1 ChIA-PET datasets obtained from female naïve hESCs (Ji et al., 2016), suggesting that these loops occur independently from the XCI status of the cells (Figure S3C).

As insulated chromatin neighborhoods have been shown to favor the communication between genes and their regulatory elements (Ji et al., 2016; Sun et al., 2019), we hypothesized that XIST/JPX longrange interactions could provide a structural framework for JPX transcription to regulate XIST expression. To test this hypothesis, we used a CRISPR inhibition strategy (CRISPRi) (Gilbert et al.,

10 2013) in female primed H9 hESCs, where three guide RNAs were used independently to recruit a catalytically inactive Cas9 fused to a KRAB co-repressor to the $J P X 5$ ' region, in order to prevent its transcription (Figure 3C). This system efficiently triggered the local deposition of the H3K9me3 repressive mark on a restricted region surrounding $J P X$ transcription start site (TSS) (Figure 3D). As a result, RNAPII recruitment to JPX TSS was compromised (Figure S3D) and transcription at the

15 locus was severely impaired; $J P X$ remained transcribed in less than $\sim 15 \%$ of the cells as assessed by RNA-FISH (Figure 3E) and JPX RNA levels were reduced by 90\% (Figure S3E). RNAPII occupancy was also decreased at the Ht1 (Figure 3F), indicating that this strategy efficiently reduced RNAPII processing along the $70 \mathrm{~kb}$ of the $J P X$ gene. In these conditions, $X I S T$ steady-state RNA levels were significantly reduced (Figure $3 \mathrm{G}$ ), as were the percentage of cells with XIST accumulation (Figure

$203 \mathrm{H}$ ) and focal enrichment of the H3K27me3 repressive mark (Figure S3F). In addition, the cells that retained XIST expression displayed smaller XIST RNA cloud compared to the control condition (Figure $3 \mathrm{H}$ ), indicating that all cells in the population were affected by JPX inhibition. As XIST repression may result from XCI erosion, we tested whether inhibition of $J P X$ transcription could favor this process. For this, we monitored the accumulation of the XACT lncRNA and the transcription of 25 the POLA1 gene from the Xi by RNA-FISH, as the expression of these genes from the Xi are early markers of XCI erosion and precedes the loss of XIST expression in H9 hESCs (Vallot et al., 2015). Inhibition of $J P X$ transcription did not trigger either XACT or POLA1 transcription from the XISTcoated Xi (Figure S3G) and the two genes remained solely expressed from the active X. Moreover, we could not link the reduction of XIST expression to perturbation of YY1 binding on XIST promoter

30 (Figure S3H), a known regulator of XIST expression in human cells (Makhlouf et al., 2014), suggesting that $J P X$ acts through YY1-independent mechanisms. We also verified that reduced XIST expression did not result from an ectopic deposition of $\mathrm{H} 3 \mathrm{~K} 9 \mathrm{me} 3$ at XIST promoter due to the CRISPRi strategy (Figure S3I). Altogether, our results, which were reproduced in another primed hESCs line (WIBR2, Figure S3J-K), demonstrate that JPX transcription is required for proper XIST expression. 
A
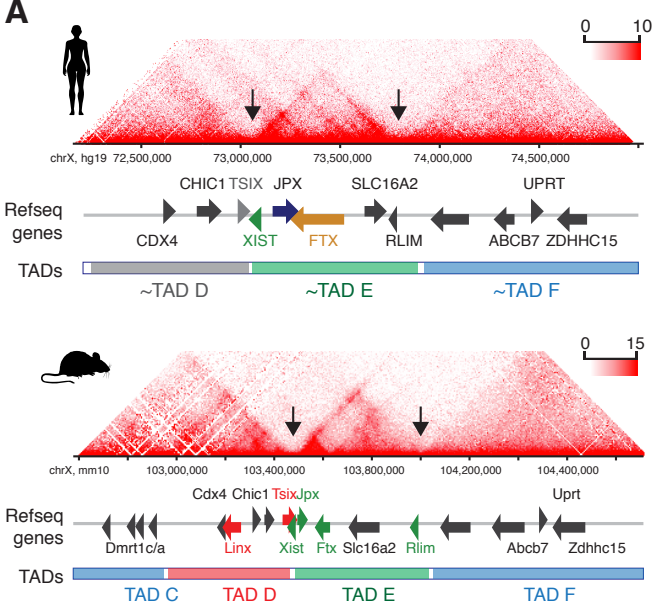

B

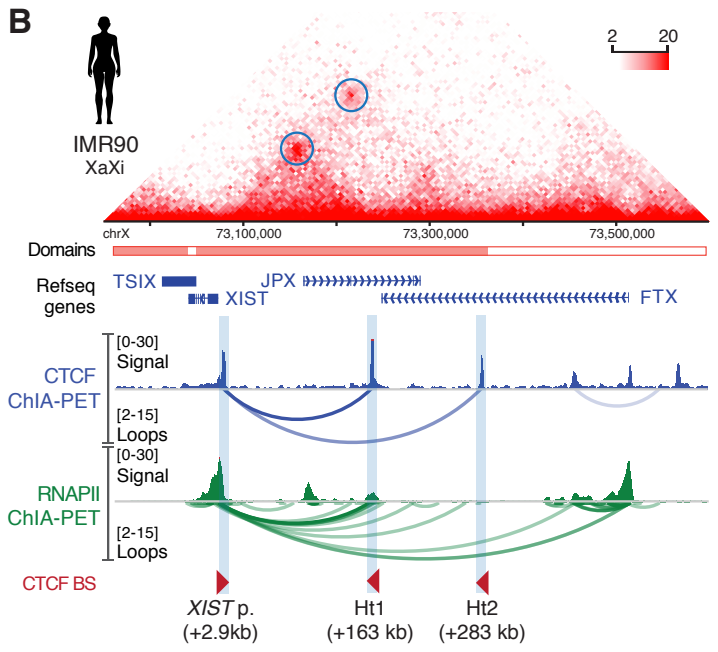

E
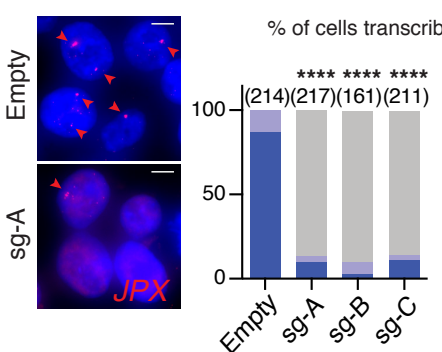

$\mathbf{F}$

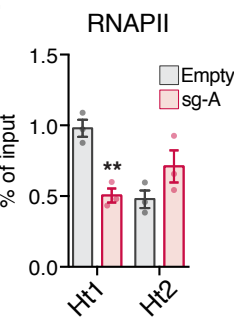

H

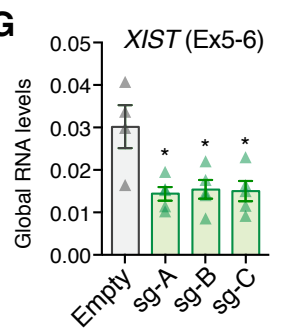

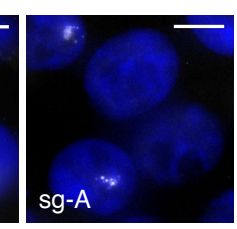

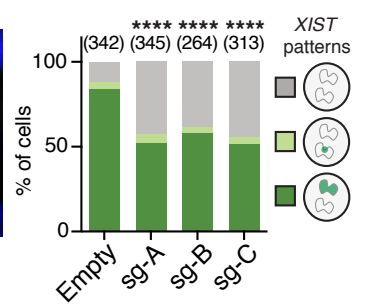

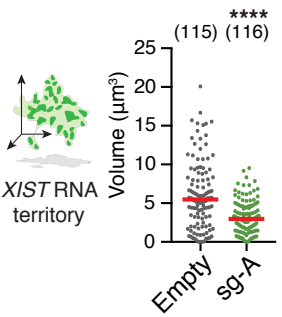

Figure 3: $X I S T$ expression requires $J P X$ transcription.

(A) The human XIC is partitioned in discrete topological domains (TAD C to F) that are syntenic to that of the mouse Xic (TAD D to F); Xist/XIST TAD E boundaries are highlighted by black arrows. HiC data from human fetal fibroblasts and mouse ESC (Bonev et al., 2017; Rao et al., 2014).

(B) XIST and JPX interact (Hi-C, IMR90) through CTCF- and RNAPII-mediated loops (ChIA-PET K562). Called loops are highlighted by blue circles. (See also Figure S3A-C).

(C-D) Scheme of CRISPRi strategy to inhibit $J P X$ transcription in primed hESCs. In this condition, a strong and local enrichment of H3K9me3 could be observed at $J P X$ promoter, ChIP-qPCR, $\mathrm{n}=4$. (See also Figure S3D).

(E) The number of cells expressing $J P X$ was strongly reduced in CRISPRi conditions, with both $J P X$ alleles being efficiently silenced one week after lentiviral infection with the guides. Left: Representative images. Right: scoring of $J P X$ RNA-FISH signals (Chi-square test). (See also Figure S3E).

(F) Inhibition of $J P X$ transcription reduced RNAPII availability at the Ht1, ChIP-qPCR, $\mathrm{n}=3$.

(G) XIST steady state RNA levels were reduced upon inhibition of $J P X$ transcription (RT-qPCR, n=4). (See also Figure S3F).

(H) JPX CRISPRi resulted in a decrease in the number of cells expressing XIST (Chi-square test) and on the volume of XIST RNA cloud (Mann-Whitney test), RNA-FISH. (See also Figures S3H-K).

Error bars represent standard deviation; n.s., not significant; *p $<0.05 ;{ }^{* *} \mathrm{p}<0.01 ; * * * \mathrm{p}<0.001$; $* * * * \mathrm{p}<0.0001$. Unpaired two-tailed $\mathrm{t}$-tests to the empty condition unless stated otherwise. Number of counted cells is in brackets. 

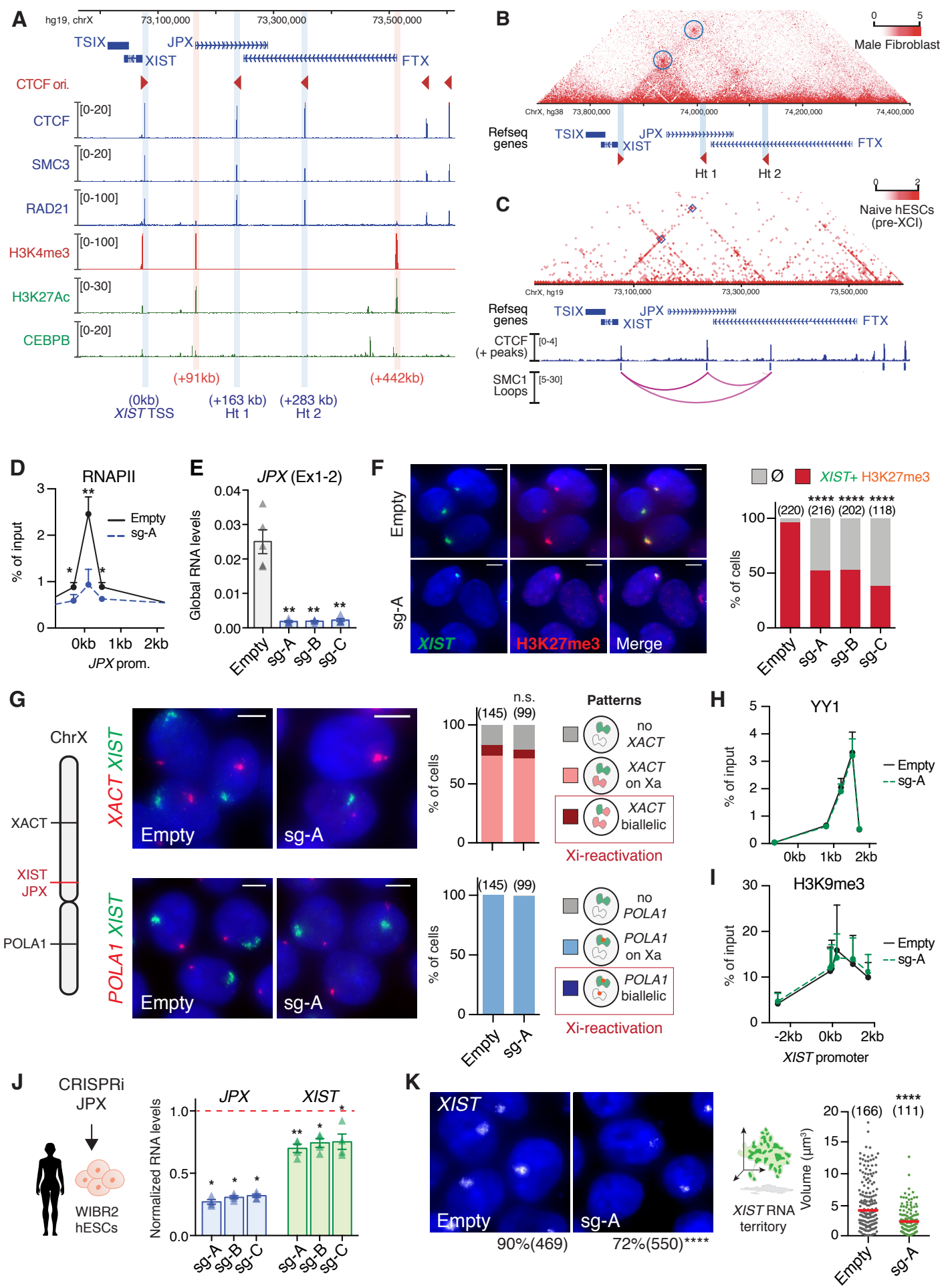

Figure S3: XIST expression requires $J P X$ transcription, related to Figure 3

(A) Within the XIST-associated TAD, CTCF binding sites are co-occupied by the cohesin complex (RAD21 and SMC3). The hotspots $(\mathrm{Ht} 1 / \mathrm{Ht} 2)$ are not enriched with enhancer-associated chromatin marks (H3K27Ac) or protein (CEBPB) in female fetal fibroblasts; active promoters in this region are highlighted by the H3K4me3 ChIP-seq track.

(B) Structural organization of XIST-associated TAD in male fibroblasts (HFF-c6; (Dekker et al., 2017).

Called loops are highlighted by blue circles.

(C) In naïve hESCs from (Ji et al., 2016), JPX and XIST are interacting through SMC1-mediated loops, which coincide with CTCF-mediated loops in Fig.2a. The heatmap represents raw SMC1 interactions and arcs represents high confidence loops. 
(D-E) $J P X$ CRISPRi prevented RNAPII recruitment at $J P X$ promoter (ChIP-qPCR, $\mathrm{n}=3$ ) and resulted in a strong decrease of $J P X$ RNA levels, RT-qPCR, $\mathrm{n}=4$.

(F) $J P X$ CRISPRi led to the simultaneous loss of XIST RNA clouds (RNA-FISH) and H3K27me3 foci (IF); right panel represents the fraction of double positive cells for XIST and H3K27me3 cells, Fischer's exact test.

(G) In contrast to XCI erosion, $J P X$ CRISPRi did not trigger $X A C T$ reactivation from the XIST-coated $\mathrm{Xi}$, as assessed by double XIST and XACT RNA-FISH (Chi-square test). Similarly, no Xi-reactivation of POLAI transcription could be identified by double XIST and POLAI RNA-FISH (Fischer's exact test). (H) $J P X$ CRISPRi did not affect YY1 binding at XIST promoter, ChIP-qPCR, $\mathrm{n}=3$.

(I) $J P X$ CRISPRi did not result in ectopic H3K9me3 enrichment at XIST promoter, ChIP-qPCR, $\mathrm{n}=4$. $(\mathrm{J}-\mathrm{K})$ Inhibition of $J P X$ transcription in WIBR2 hESC induced a decrease in XIST RNA levels (RT-qPCR, unpaired two-tailed t-test, $\mathrm{n}=3$ ), in the number of cells expressing XIST (Fischer's exact test) and in the volume of XIST RNA cloud (Mann-Whitney test) - red bars: median.

Error bars represent standard deviation; n.s., not significant; ${ }^{*} \mathrm{p}<0.05 ;{ }^{* *} \mathrm{p}<0.01 ; * * * \mathrm{p}<0.001$; $* * * * \mathrm{p}<0.0001$. Unpaired two-tailed t-tests to the empty condition unless stated otherwise. Number of counted cells is in brackets. 


\section{XIST expression requires a functional $J P X$ allele in cis.}

To further explore the contribution of $J P X$ transcription to XIST regulation, we generated deletions of $J P X$ promoter region in primed hESCs using the CRISPR-Cas9 technology. Based on $J P X$ promoter features and its expression in $\mathrm{H} 9$ cells, we designed guides RNAs to delete a $\sim 7 \mathrm{~kb}$ region encompassing the three first exons of $J P X$ (Figure S4A). We developed a strategy where hESCs cotransfected with two sgRNAs, each targeting a region upstream and downstream of $J P X$ TSS, could be selected based on the expression of a fluorescent gene, GFP and mCherry, respectively (Figure S4B). Therefore, FACS-sorting of double positive cells maximizes the probability to obtain clones with a direct deletion of $J P X$ promoter. This approach allowed us to interrogate the contribution of $J P X$ transcription to XIST regulation in an allele-specific manner, and tease apart cis- from transeffects of the LRG (Figure 4A). However, as no SNP could be identified in H9 cells within the deleted region, we systematically performed simultaneous $J P X$ and $A T R X$ RNA-FISH to determine on which of the two X-chromosomes (Xa or Xi) $J P X$ was still transcribed. Using this strategy, we selected two clones carrying heterozygous deletions of $J P X$ promoter region for further investigation, in which the deletion occurred either on the active $\mathrm{X}$-chromosome $(\triangle J P X-\mathrm{Xa})$ or on the inactive $\mathrm{X}(\triangle J P X$-Xi) (Figure 4B and S4C-D). Interestingly, the two clones displayed different JPX RNA levels depending of the deleted allele, with the expression level in $\triangle J P X$-Xa clone reaching only $\sim 15 \%$ of that of the WT clone, and $\sim 80 \%$ in the $\triangle J P X-\mathrm{Xi}$ clone (Figure $4 \mathrm{C}$ ). This is in agreement with $J P X$ being predominantly expressed from the Xa in WT cells, as found for most genes that escape XCI (Carrel et al., 1999). Remarkably, we found that XIST expression was perturbed exclusively in the $\triangle J P X-\mathrm{Xi}$ clone with RNA; XIST RNA levels were reduced by half compared to the WT and $\triangle J P X$-Xa clone (Figure 4D) with only $\sim 55 \%$ of the cells displaying XIST RNA accumulation (Figure 4E) and H3K27me3 foci (Figure S4E). Moreover, XIST RNA territory in the remaining XIST-positive cells were significantly smaller in the $\triangle J P X-\mathrm{Xi}$ clone (Figure $4 \mathrm{~F}$ ), indicating that $X I S T$ expression was also impacted in those cells. Altogether these results show that transcription originating from the $J P X$ promoter region is required to sustain XIST expression in cis in human post-XCI cells. Moreover, the fact that $J P X$ RNA levels were the least perturbed in the $\triangle J P X$-Xi clone (Figure $4 \mathrm{C}$ ) confirmed that $J P X$ RNA does not control XIST expression in human.

As $J P X$ induction seems to precede $X I S T$ upregulation during human pre-implantation development, we investigated whether its transcription could be important for the de novo induction of XIST expression. To do so, we proceeded to the chemical resetting of the primed $\triangle J P X$ and WT lines into the naïve state of pluripotency (Guo et al., 2017). As a control, we also converted fully XIST-negative eroded primed hESCs to ensure that the resetting process could efficiently trigger XIST upregulation 
and X-chromosome reactivation (XCR) as observed with this method (Guo et al., 2017) and others (Sahakyan et al., 2017; Theunissen et al., 2016; Vallot et al., 2017). After 7 passages ( 45 days) in naïve culture medium, all cell lines displayed dome-shaped colonies (Figure S4F) and proper induction of key naïve-specific pluripotency markers (Figure 4G) such as the transcription factors KLF4 and LBP9 (Takashima et al., 2014), indicating an efficient transition to the naïve-like state. $\mathrm{XCR}$ was observed to same extent in all cell lines, as inferred from the bi-allelic transcription of ATRX ( 75-85\%, Figure $4 \mathrm{H})$, which confirmed that XCR occurred independently from the XISTexpressing status of the parental primed hESCs (Sahakyan et al., 2017). XCR could also be detected at the level of XIC-linked genes, notably by the biallelic transcription of the Xa-specific FTX gene

10 (Figure $\mathrm{S} 4 \mathrm{G}$ ) and for $J P X$, which is more expressed in naïve $\triangle J P X$-Xa cells compared to primed (Figure 4I). We found that XIST was strongly reactivated upon the resetting of the eroded cells (Figure $4 \mathrm{~J}$ ), with the proportion of naïve XIST-expressing cells reaching about 95\%, as assessed by RNAFISH (Figure 4K). In agreement with previous studies, XIST accumulation remained mostly monoallelic and likely confined to the former $\mathrm{Xi}$, with less than $3 \%$ of XaXa cells displaying biallelic $X I S T$ RNA clouds. Resetting of XIST-expressing cells (WT and $\triangle J P X$-Xa clones) did not increase the proportion of biallelic XIST RNA clouds and XIST RNA clouds remained associated to the former Xi (Sahakyan et al., 2017). By contrast, XIST reactivation could not be observed in cells where $J P X$ is deleted on the $\mathrm{Xi}$ allele, in which XIST expression remained low and restricted to an even lower percentage of cells, compared to primed conditions (38\% naïve vs. $58 \%$ primed, Figure $4 \mathrm{~K}$ ). Deletion of the JPX promoter region thus prevents de novo XIST upregulation in cis during conversion of primed to naïve human ESCs. These analyses altogether suggest that $J P X$ transcription is required for proper XIST upregulation in cis during early human pre-implantation development.

\section{$J p x$ RNA regulates $X I S T$ expression in mouse post-XCI cells.}

25 Previous studies have shown that the deletion of a single allele of the Jpx gene or shRNA-mediated knockdown of Jpx is sufficient to prevent Xist upregulation (Carmona et al., 2018; Tian et al., 2010), with Jpx acting through RNA-based mechanisms, both in cis and in trans. Considering the discrepancy with the results we obtained in human, we decided to revisit the function of Jpx RNA in the mouse, by rigorously matching both our experimental approaches and the cellular models. We

30 performed LGs-mediated KD experiment in mouse embryonic fibroblasts (pMEFs) and murine epiblast-derived stem cells (EpiSCs). Murine EpiSCs share similarities with primed hESCs in terms of transcriptional signatures, signaling pathways and XCI status (Brons et al., 2007; Tesar et al., 2007), while pMEFs parallel the primary fibroblasts of fetal origin used in this study (Figure 2C-H and S2F-G). Both cell types display one inactive X-chromosome coated by Xist and express Jpx. First, we investigated mouse $J p x$ RNA function in primary mouse embryonic fibroblasts (pMEFs) 
A

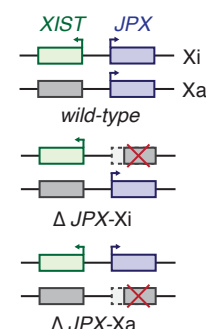

B

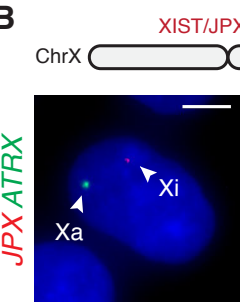

$\triangle J P X-X a$

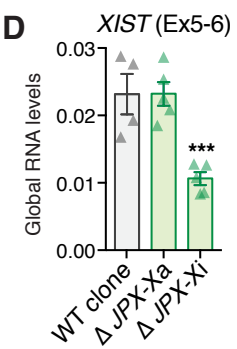

E
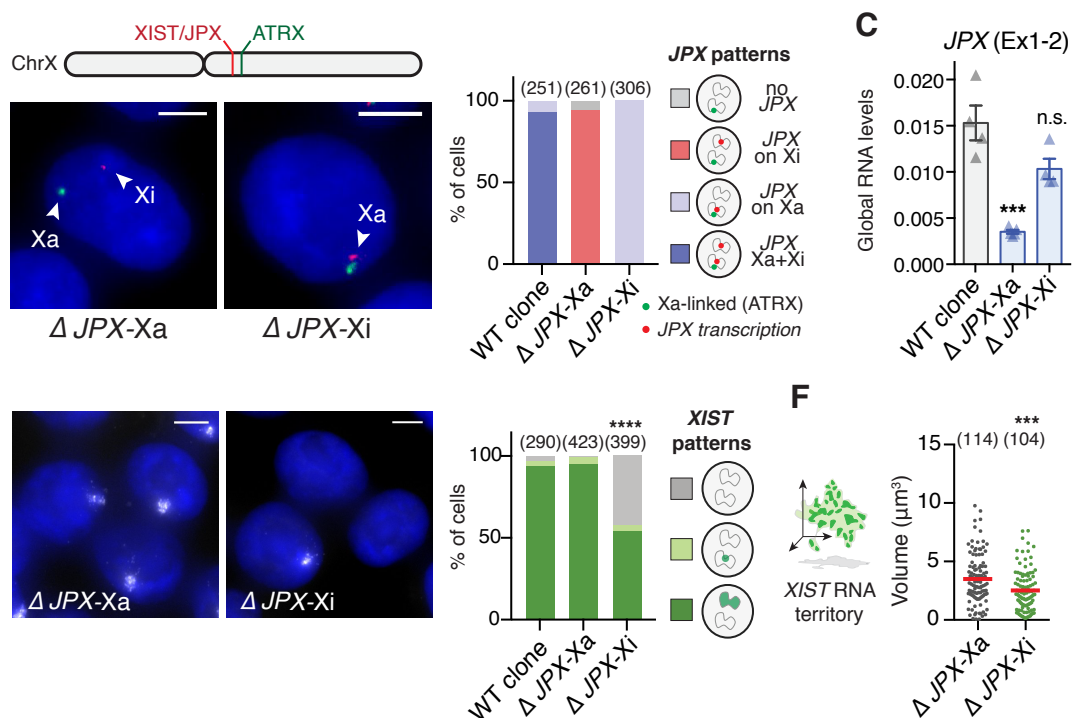

G
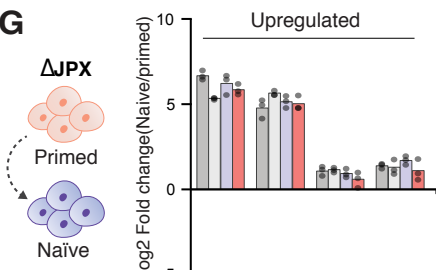

.

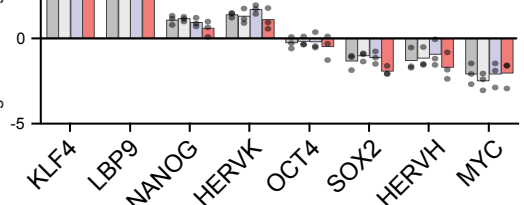

$\square$ Clone WT

$\square X I S T(-)$

$\square \triangle J P X X \mathrm{Xa}$

$\square \triangle J P X X \mathrm{X}$

H
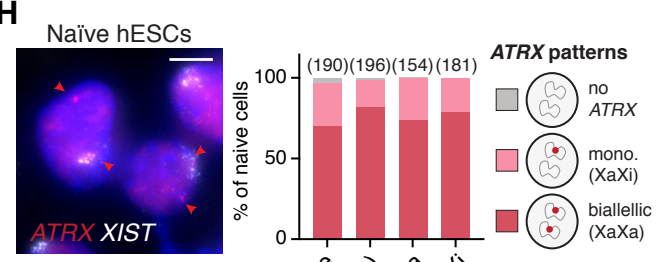

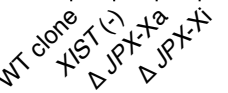
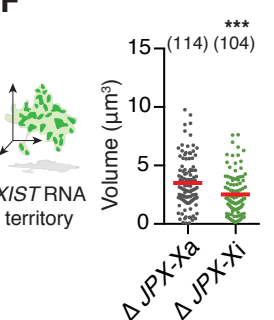

I

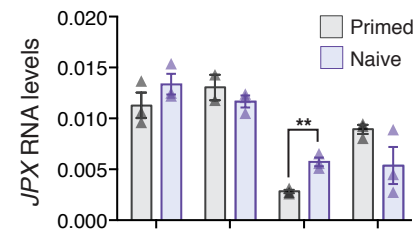

J

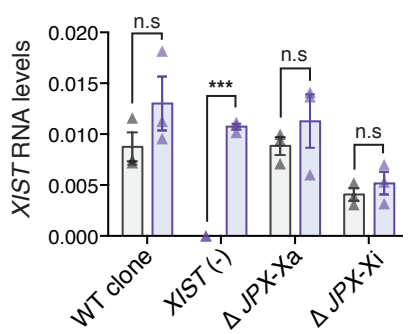

K
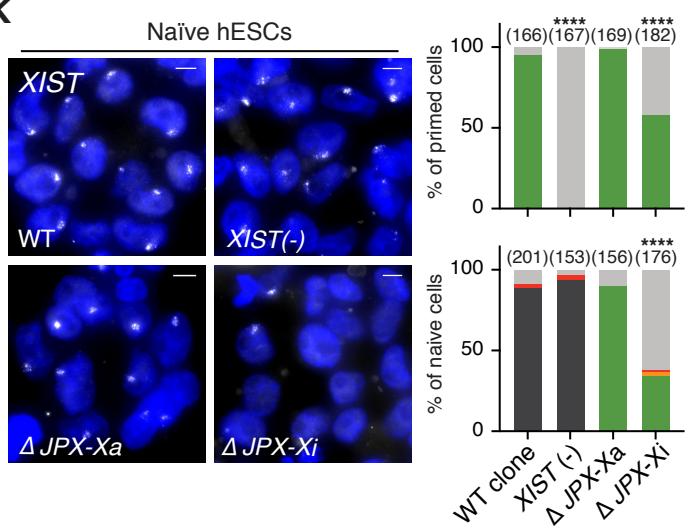

Figure 4: XIST expression requires a functional $J P X$ allele in cis.

(A) Schematic representation of WT and $J P X$-deleted hESC clones. (See also Figure S4A and S4B)

(B) Determination of the $J P X$-targeted allele by simultaneous JPXIATRX RNA-FISH. ATRX is transcribed exclusively from the $\mathrm{Xa}$ in primed hESCs. (See also Figure S4C and S4D).

(C) $\triangle J P X$-Xa cells displayed a $\sim 80 \%$ reduction of $J P X$ RNA levels compared to WT, while a moderate decrease was observed in $\triangle J P X$-Xi cells ( 20\%), suggesting an asymmetric expression of $J P X$ from the two X chromosomes, RT-qPCR, $\mathrm{n}=4$.

(D) XIST steady states RNA levels were reduced when JPX promoter was deleted in cis ( $\triangle J P X-\mathrm{Xi})$, but not in trans $(\triangle J P X-\mathrm{Xa}), \mathrm{RT}-\mathrm{qPCR}, \mathrm{n}=4$.

(E-F) In $\triangle J P X$-Xi cells, both the number of cells expressing XIST (Chi-square test) and the volume of XIST RNA cloud (Mann-Whitney test) were reduced, RNA-FISH. (See also Figure S4E).

(G) Average $\log 2$ fold-change of transcript levels between the naïve and the parental primed hESC clones for a selection of markers, RT-qPCR, $n=3$. (See also Figure S4F).

(H) The Xi was properly reactivated in more than $70 \%$ of cells in the different cell lines based on biallelic expression of $A T R X$, RNA-FISH. (See also Figure S4G). 
A

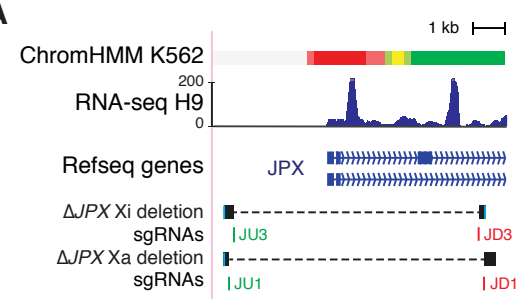

D

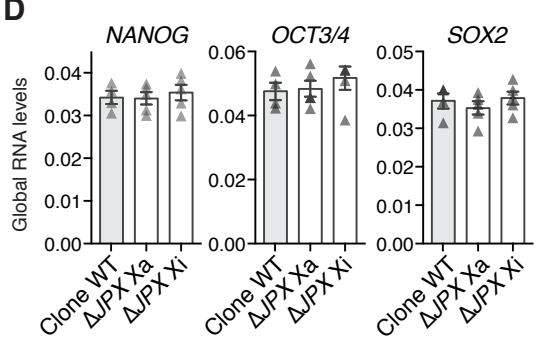

B

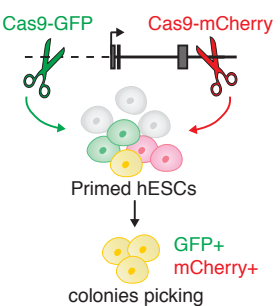

E
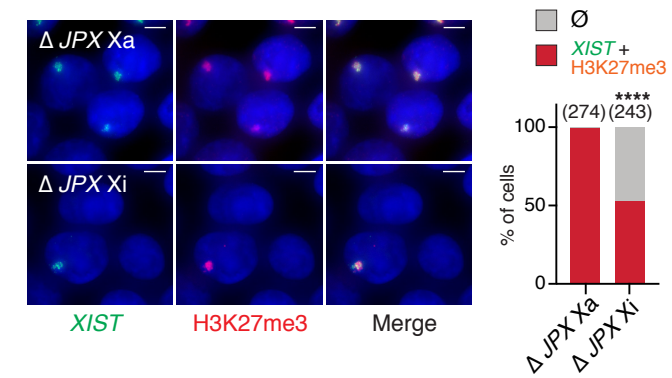

$\mathbf{F}$
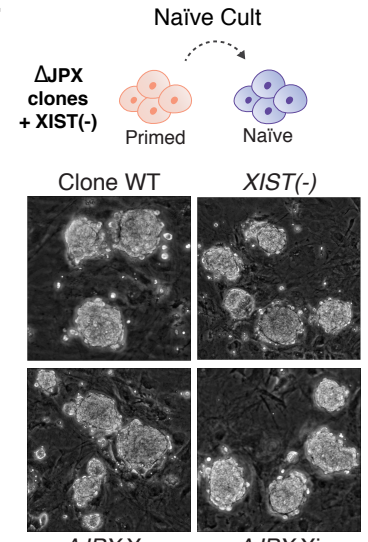

$\triangle J P X \times a$

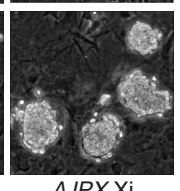

G

홈
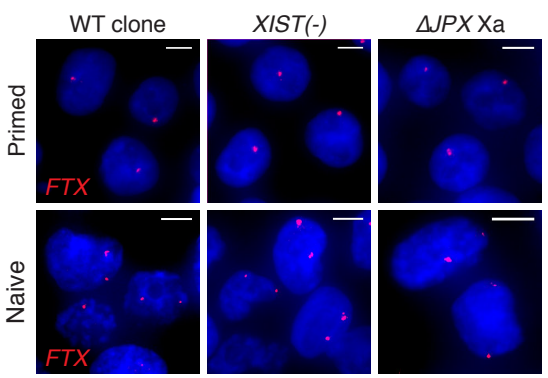

$\triangle J P X X \mathrm{i}$
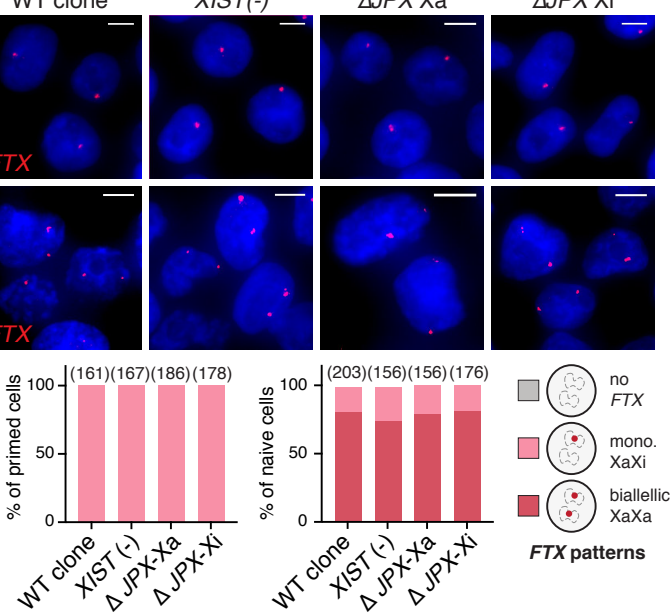

Figure S4: XIST expression requires a functional JPX allele in cis, related to Figure 4

(A) Map of the $J P X 5$ ' region, with various promoter features indicated, such as the chromatin (chromHMM) and transcriptional states (H9 RNA-seq, H3K4me3). The bottom tracks show the guides position (in green, guides coupled to a Cas9-GFP; in red guides coupled to a Cas9-mCherry) and sequence alignments spanning the deleted region to the reference genome in the two clones.

(B) Scheme of the strategy to produce genomic deletion using the CRISPR-Cas9 system coupled to FACssorting in hESCs.

(C) PCR-genotyping of the two heterozygote clones obtained.

(D) $J P X$ deletion did not impact on the steady state RNA levels of key pluripotency markers, RT-qPCR, error bars represent standard deviation, $\mathrm{n}=4$.

(E) Deletion of $J P X$ promoter on the $\mathrm{Xi}$, but not the $\mathrm{Xa}$, led to a decrease in the percentage of cells with XIST RNA clouds and H3K27me3 foci (Immunofluorescence) (Fischer's exact test). P-value: $<0.0001$ $(* * * *)$.

(F) Resetting primed hESCs to the naïve state results in similar colonies with dome-shaped appearance, independently of the genotype.

(G) Analysis of FTX gene expression by RNA-FISH showed robust biallelic expression following resetting of primed into naïve hESCs.

Scale bars are $5 \mu \mathrm{m}$. Number of counted cells is in brackets. 
derived from 13.5 days post-coitum mouse embryos using three distinct LNA Gapmers targeting different exons, to minimize the probability of random off-target effects (Stojic et al., 2018) (Figure 5A). This approach was efficient in depleting $J p x$ RNA (Figure 5B), and the strongest effect was obtained with the mLG1, which is expected to target all Jpx RNA isoforms. The three LGs induced a decrease in spliced Xist RNA levels (Figure 5C) that correlated with the extent of Jpx RNA depletion (Pearson correlation $=0.96$, p-val. $=0.035)$, suggesting a dose-dependent effect of Jpx RNA. RNAFISH analyses revealed that most of the cells were affected by Jpx KD, as both the percentage of Xist positive cells and the volume of the remaining Xist RNA clouds were reduced (Figure 5D). As previously, we verified that the observed effects of Jpx mLGs were not due to transcriptional

10 inhibition through quantification of Jpx nascent transcripts by RNA-FISH and after ethynyl uridine (EU) incorporation followed by pull-down (Figure S5A-B). We could thus conclude that the observed Xist downregulation can be attributed to the depletion of Jpx mature transcripts only, and not to alterations of Jpx ongoing transcription. Similarly to pMEFs, depletion of Jpx RNA in EpiSCs led to a decrease in Xist RNA levels (Figure 5E), without ectopic expression of Xist negative regulators in

15 the mouse, namely its antisense Tsix (Figure 5F) or the pluripotency factors REX1 and KLF4 (Figure 5G) (Navarro et al., 2010). These data demonstrate the contribution of mouse Jpx RNA to the maintenance of Xist expression in post-XCI cells and confirm the role of Jpx as a potent regulator of Xist.

\section{Mechanisms of XIST regulation by $J P X$ have diversified during evolution}

To further decipher the mechanisms underlying the different molecular function of $J P X$, we investigated which step of Xist/XIST biogenesis was under the control of the Jpx/JPX LRG in mouse and human. Since previous work reported that Jpx RNA could activate Xist by evicting the CTCF protein from its TSS (Sun et al., 2013), we investigated CTCF binding profile across the Xist promoter upon Jpx

$25 \mathrm{KD}$ in pMEFs. Binding of CTCF to a position $\sim 1 \mathrm{~kb}$ upstream of Xist TSS was significantly increased upon Jpx KD (Figure 6A), an effect we also observed on the imprinting control region of H19 (Figure S6A). Nevertheless, we probed the impact of this change of CTCF binding on Xist transcription by measuring the level of Xist premature transcripts. Quite unexpectedly, we could not detect changes in Xist premature transcript, suggesting that transcription was unaffected by Jpx KD (Figure 6B). To confirm this, we performed nascent RNAs pulldown (Figure 6C) and single-cell level by RNA-FISH using stranded oligo-probes detecting Xist first intron (Figure 6D). While these approaches were suitable to detect Xist transcriptional changes upon $Y y 1 \mathrm{KD}$, a known regulator of Xist transcription (Figure 6C-D and Figure S6B-E) (Makhlouf et al., 2014), we could not detect any transcriptional deregulation upon Jpx KD. Altogether, these data demonstrate that Jpx RNA acts downstream of Xist transcription and is required for proper Xist RNA accumulation. 
A

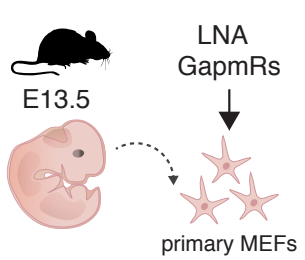

B

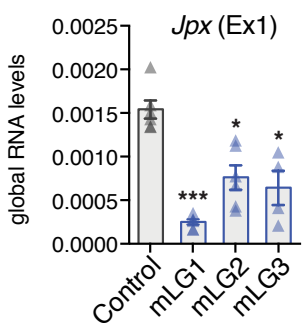

mouse Jpx RNAs

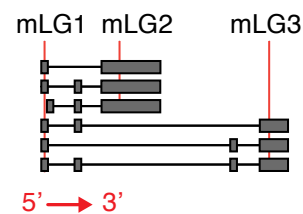

C

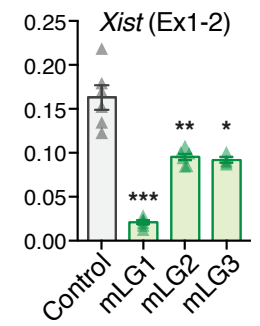

D
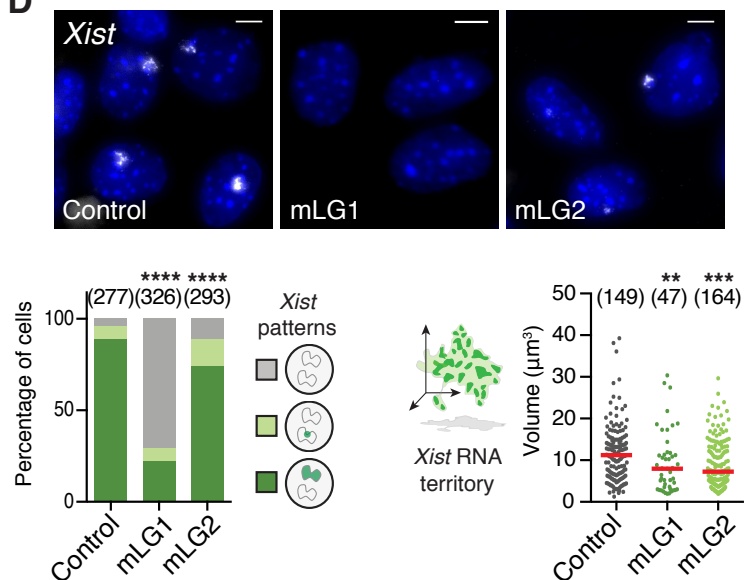

E
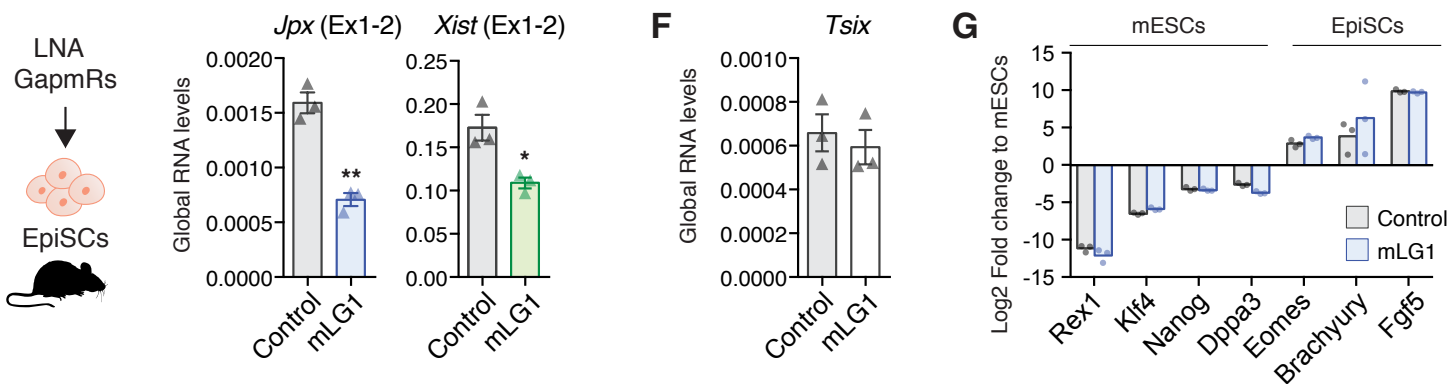

Figure 5: Jpx RNA regulates XIST expression in mouse post-XCI cells.

(A) Schematic representation of LNA GapmeRs (LG) lipofection in primary MEFs; LGs-targeted regions (red lines) are indicated on Jpx RNA isoforms.

(B-C) LG-transfected pMEFs showed reduced Jpx and Xist RNA levels, RT-qPCR; n=4.

(D) Jpx KD reduced the number of Xist expressing cells (Chi-square test) and the volume of Xist RNA cloud (Mann-Whitney test), RNA-FISH. Red bars: median.

(E) In EpiSCs, Jpx KD led to a decrease in Xist RNA levels, RT-qPCR, $\mathrm{n}=3$.

(F) Tsix is not re-expressed in EpiSC transfected with Jpx-targeting LGs.

(G) $\log 2$ expression fold change for a selection of markers (Brons et al., 2007; Tesar et al., 2007) in EpiSC transfected with control or $J p x$-targeting LG, normalized to expression in mESC, RT-qPCR, $\mathrm{n}=3$.

Error bars represent standard deviation; n.s., not significant; *p $<0.05 ;{ }^{* *} \mathrm{p}<0.01 ; * * \mathrm{p}<0.001$;

$* * * * \mathrm{p}<0.0001$. Unpaired two-tailed t-tests to the control LG unless stated otherwise.

See also Figure S5. 
A
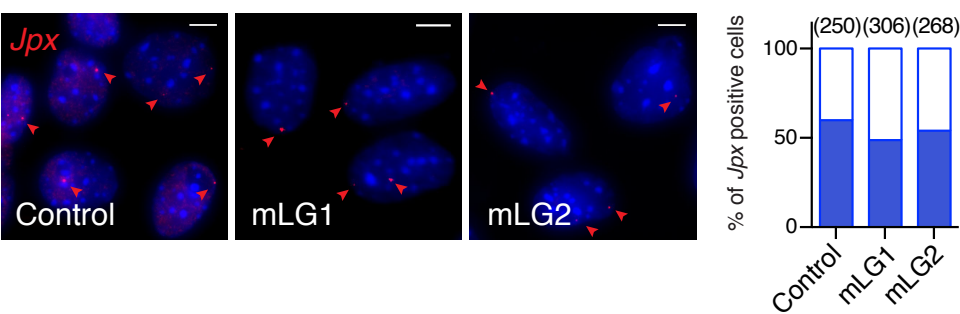

B

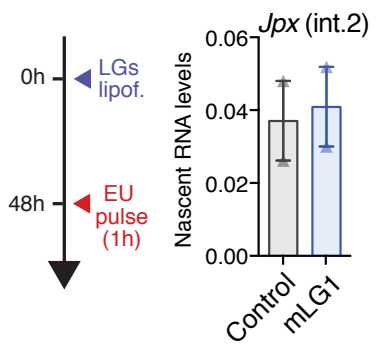

Figure S5: Jpx RNA regulates XIST expression in mouse post-XCI cells, related to Figure 5 (A) RNA-FISH of $J p x$ performed $48 \mathrm{~h}$ after LGs lipofection in pMEFs. Left panel: representative images. Right panel: quantification of $J p x$ positive cells (blue fill). Number of counted cells is in brackets. (B) Nascent RNA pulldown of EU-labelled nascent transcripts experimental scheme and Jpx RNA quantification, RT-qPCR, n=2. Error bars represent standard deviation. 
In striking contrast, XIST unspliced RNA levels were strongly decreased following inhibition of human $J P X$ transcription, independently of CTCF binding changes (Figure 6E-F). This was further supported by the observed reduction of phospho-Ser5 RNAPII recruitment at XIST promoter (Figure 5c). Severe impairment of XIST ongoing transcription following JPX transcriptional inhibition was also evident at the single-cell level, when stranded oligo-FISH probes were used to detect human $X I S T$ intronic regions (Figure 6H). This suggests a transcriptional crosstalk between JPX and XIST in human, where ongoing transcription across the JPX locus would favor the recruitment of the transcription machinery at XIST, possibly through local 3D interaction and chromosomal looping. Our results therefore demonstrate that not only the functional module of $J p x / J P X$ differs between human and mouse, but also their mode of action to regulate Xist/XIST.

\section{DISCUSSION}

Here, we interrogated the XIST regulatory network in human early development, and the extent to which regulatory networks essentially based on LRGs operate similarly in different species. Through unbiased analysis of expression dynamics of XIC-linked genes, we identified JPX as the best candidate for promoting XIST activation, and through functional investigation in various human contexts, we demonstrated a major and ubiquitous role for JPX in XIST expression. Doing so, we provided the first evidence that resetting human primed to naïve pluripotent stem cell may constitute a system of choice to study the regulatory network at stake for post-fertilization XIST activation in human. We further determined that transcription across $J P X$ is important in this process and that $J P X$ RNA is not involved in XIST regulation in human cells. Furthermore, JPX acts in cis to promote XIST transcription, while we show that Jpx RNA acts downstream of Xist transcription on Xist metabolism in the mouse. The choice of cellular models was critical for the comparative analysis of $J P X / J p x$ mode of action in human and mouse. Indeed the early steps of XCI differs markedly in these species, with initial XIST up-regulation being uncoupled from XCI in human, and, more importantly, not currently recapitulated in any ex vivo model. We therefore chose to focus most of our investigation on the maintenance of XIST expression, for which comparable cellular systems were available in the two species studied. The reproducibility of the results in two post-XCI contexts, primed pluripotent stem cells and fetal fibroblasts, strengthens our conclusions and provide the first evidence that an LRG from the XIC plays similar function in different mammalian species but operates via different mechanisms. Jpx/JPX therefore stands as a key component of the Xist/XIST regulatory network in mouse and human. 
A

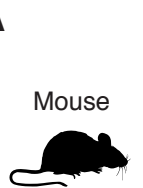

D

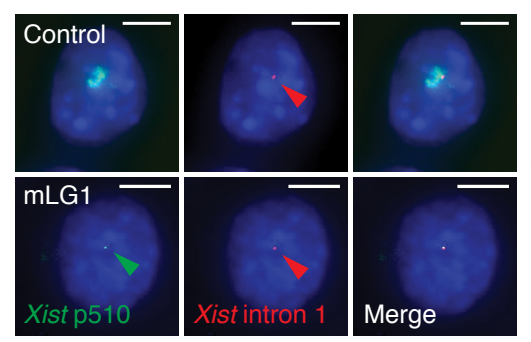

E

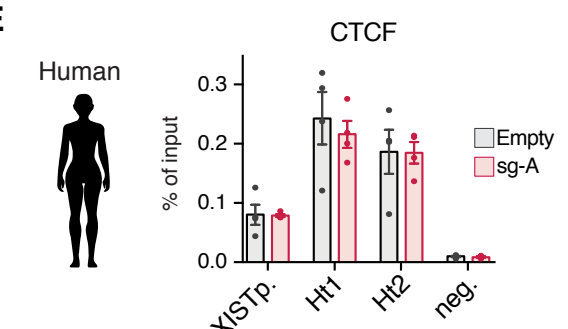

H

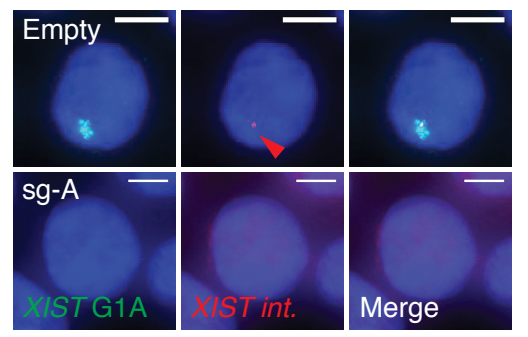

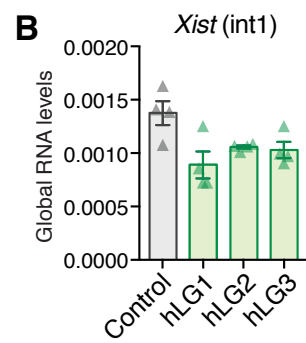

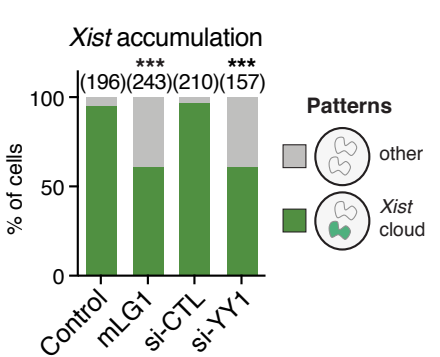

$\mathbf{F}$
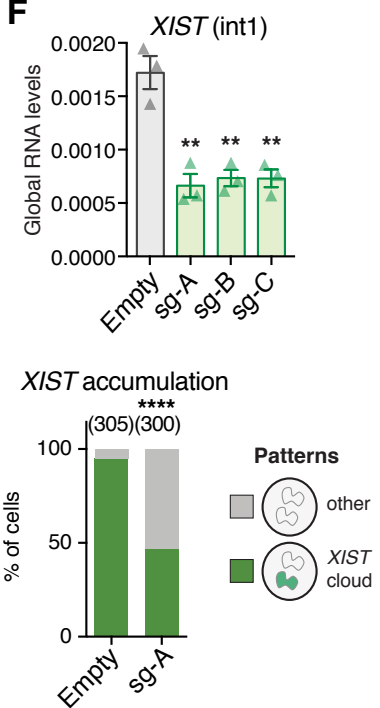

C
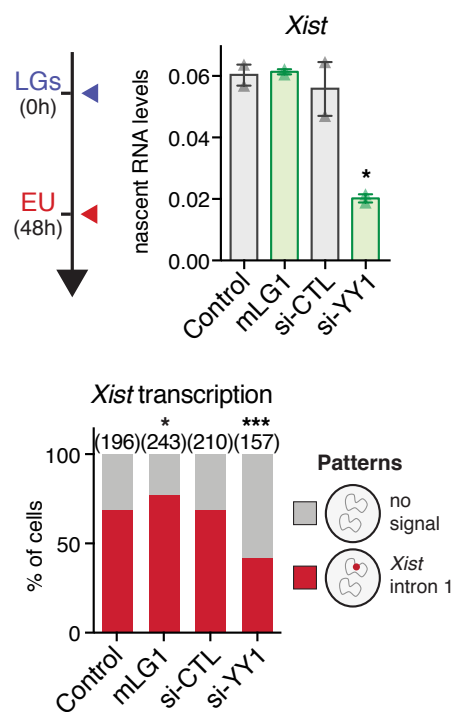

G
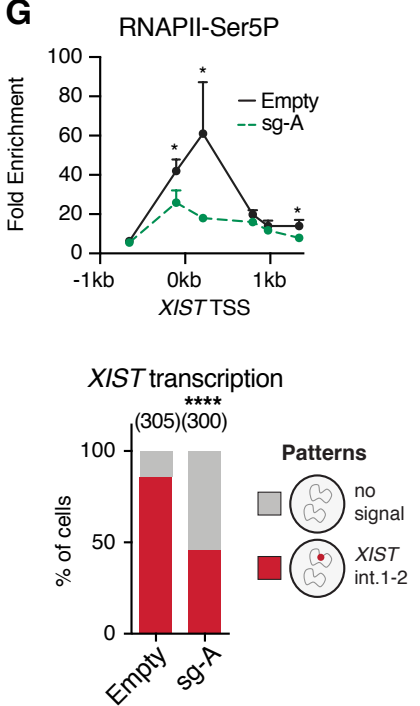

Figure 6: Mechanisms of XIST regulation by $J P X$ have diversified during evolution.

(A) In the mouse, CTCF binding to Xist proximal promoter region is increased upon Jpx KD in pMEF, ChIP-qPCR, n=3. (see also Figure S6A)

(B) KD of Jpx RNA did not impact on Xist premature transcript levels, intronic RT-qPCR, $\mathrm{n}=3$ (See also Figure S6B-D).

(C) Xist transcription was affected by $Y y 1 \mathrm{KD}$, but not by $J p x \mathrm{KD}$, in pMEFs when quantified after pulldown of EU-labelled nascent transcripts, RT-qPCR, $\mathrm{n}=3$.

(D) Jpx KD did not affect Xist ongoing transcription (intron 1 stranded oligo-FISH probes) but only on its accumulation (p510 probe) (Fisher's exact test). $Y y 1 \mathrm{KD}$ affects both transcription and accumulation of Xist. (See also Figure S6E)

(E) In human, JPX CRISPRi did not affect CTCF enrichment at XIST promoter or at both interaction hotspots, ChIP-qPCR, $\mathrm{n}=3$.

(F) XIST premature transcripts levels are reduced following JPX CRISPRi, intronic RT-qPCR, $\mathrm{n}=3$.

(G) Inhibition of $J P X$ transcription prevented RNAPII (CTD-phospho-Serine5) recruitment at XIST promoter, ChIP-qPCR, $\mathrm{n}=4$.

(H) The number of cells with XIST RNA accumulation (G1A probe) and transcription (intronic stranded oligo-FISH probes) is reduced following $J P X$ CRISPRi.

Error bars represent standard deviation; ${ }^{*} \mathrm{p}<0.05 ;{ }^{*} \mathrm{p}<0.01 ; * * * \mathrm{p}<0.001 ; * * * * \mathrm{p}<0.0001$. Unpaired twotailed t-tests to control condition unless stated otherwise. 

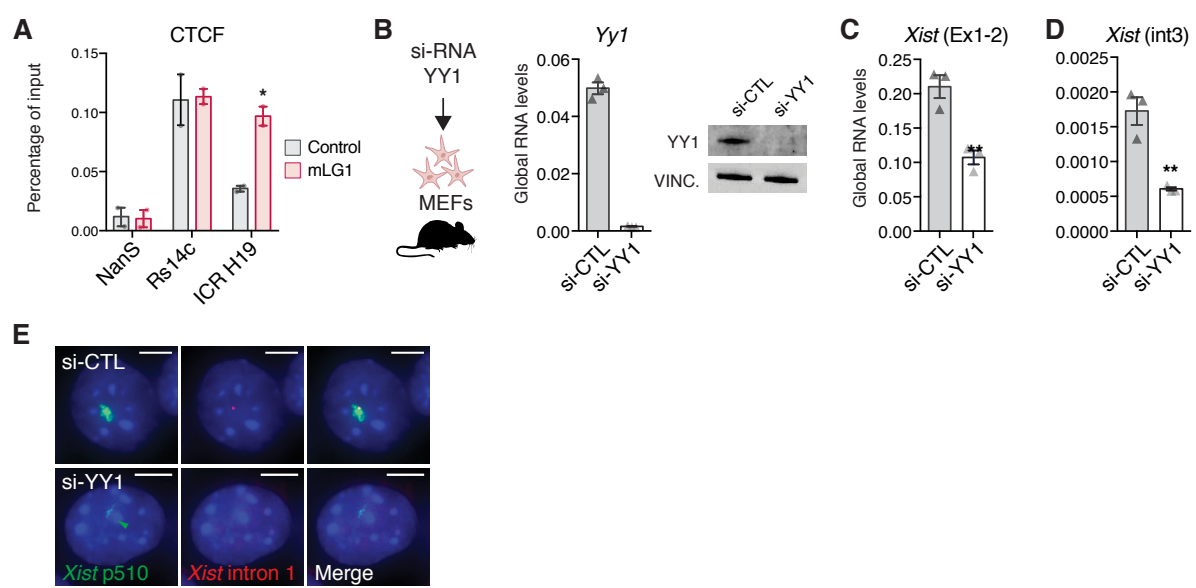

Figure S6: Mechanisms of XIST regulation by $J P X$ have diversified during evolution, related to Figure 6

(A) CTCF binding at different control positions upon LNA GapmeR transfection. Rs14c and NanS represent respectively positive and negative positions for CTCF binding, ChIP-qPCR, $\mathrm{n}=3$.

(B) Expression of $Y y 1 \mathrm{mRNA}$ and protein levels following siRNA transfection as in (Makhlouf et al., 2014).

(C-D) Expression levels of Xist mature and premature RNA following siRNA transfection.

(E) Representative images of the effect of $Y y 1$ depletion on Xist accumulation and transcription, RNAFISH using a probe covering Xist locus or intronic probes. Scale bars: $5 \mu \mathrm{m}$.

Error bars represent standard deviation; unpaired two-tailed t-test, ${ }^{*} \mathrm{p}<0.05 ; * * \mathrm{p}<0.01$. 
Addressing the functional conservation of LRGs is a challenge given their fast evolutionary rate. Overexpression of human $J P X$ RNA in trans was recently shown to complement heterozygous deletion of mouse Jpx during the establishment of XCI, suggesting that the human RNA might be functional in an ectopic context (Karner et al., 2019). Such rescue experiments using orthologous LRGs, as opposed to our strategy to tackle the role of mouse and human $J p x / J P X$ in the respective species, reveal the effect of the environment on LRG mode of action but do not interrogate LRGs' function in their endogenous contexts. The lack of XIST deregulation upon JPX RNA depletion in all human cellular contexts that we tested, together with the fact that deleting JPX impacts on XIST only in cis strongly argues against a major role for JPX RNA in trans during human XCI.

The mechanistic diversification we observed between mouse and human might be a consequence of the changes within the chromatin neighborhood encompassing the JPX locus in human. For instance, our previous work suggested that transcriptional activity of Xist in the mouse is, at least partially, regulated in cis by transcription of the neighboring Ftx LRG, independently of the Ftx RNA products

15 (Furlan et al., 2018). Interestingly, Ftx is located $141 \mathrm{~kb}$ upstream of Xist, which is comparable to the distance bridging XIST promoter to the interaction hotspot Ht1 $(\sim 163 \mathrm{~kb})$ within the human $J P X$, and interacts through CTCF-mediated loops with Xist (Furlan et al., 2018). It is within this insulated chromatin neighborhood, which has been reshaped between mouse and human, that constraints on the JPX locus might have favored diversification of JPX mode of action on XIST. One compelling 20 hypothesis from this model is that XIST transcriptional cis-regulators in eutherian species could have been co-opted based on features such as linear distance from XIST promoter, local 3D organization and XCI escaping profile. Importantly, this scenario is reminiscent of what has been observed for enhancer evolution (Villar et al., 2015), and is thus likely not restricted to $J P X$ evolution but may apply to other orthologous LRGs.

Finally, what our study provides is a proof of concept that orthologues may act differently in various species, thus epitomizing the mechanistic plasticity of LRGs through evolution. Diversification of LRGs across evolution could confer molecular drift to developmental processes, contribute to species adaptability and fitness: their strong turnover offers a plausible mechanism for generating phenotypic 30 diversity in the control of gene expression across evolution. One major challenge is the systematic identification of versatile LRGs. Indeed, both LRGs functionality, if any, and their mechanism of action are hardly predictable based on the DNA sequence alone. Like for JPX, syntenic LRGs often display strong primary sequence turnover during evolution, even among closely related species (Hezroni et al., 2015; Necsulea et al., 2014; Ulitsky et al., 2011; Washietl et al., 2014). The impact of such turnover on LRGs functional conservation is still poorly understood. In rare studies where 
the functional conservation of lncRNA molecules has been addressed, orthologues display short patches of conserved sequence that are necessary, but not sufficient, for their function (Lin et al., 2014; Ulitsky et al., 2011). This contrasts sharply with the evolutionary stability of protein-coding genes and has often raised controversies about LRGs functionality. Our study pave the way for

5 systematic experimental investigations of LRGs functional conservation with the aim to provide a definitive understanding of underlying rules. Whether mechanistic or functional, this plasticity appears to be an essential parameter to take into consideration in the context of animal modelling of human diseases involving LRGs. 
Data and materials availability: All data generated or analyzed during this study are included in the published article (and its supplementary information).

Acknowledgments: We thank lab members for critical evaluation of the work leading to this publication. We also thank Antonin Morillon, Pierre-Antoine Defossez, Claire Francastel, Jonathan Weitzman and Céline Morey for critical reading of the manuscript. We thank the Epigenomic, the Microscopy, the Vectorology and the Bioinformatics Platforms, all hosted in UMR7216 Epigenetic and Cell Fate, for technical advices and access to instruments. We acknowledge the ImagoSeine core facility of the Institut Jacques Monod, member of the France BioImaging (ANR-10-INBS-04) and the support of the Region Île-de-France (E539). The research leading to these results has received funding from the European Commission Network of Excellence EpiGeneSys (HEALTH-F4-2010257082, to C.R. and P.J.R.-G.), from the Agence Nationale pour la Recherche (ANR-14-CE10-0017,

15 to C.R.) and from the Ligue Nationale contre le Cancer (to C.R.). This study was supported by the LabEx "Who Am I?" (ANR-11-LABX-0071) and the Université de Paris IdEx (ANR-18-IDEX0001) funded by the French Government through its "Investments for the Future" program. O.R. is supported by fellowships from the French Ministry of Education and Research and from the French Medical Research Foundation (FRM). P.J.R.-G. and A.J.C. are supported by the Biotechnology and Biological Sciences Research Council (BB/M022285/1 and BB/P013406/1) and the Medical Research Council (MR/J003808/1).

Author contributions: O.R., J.-F.O. and C.R. conceived the project and planned the experiments. O.R., C.H., M.C. and J.-F.O. performed the experiments. A.J.C. and P.J.R.-G. performed LGs experiment on naïve hESCs. O.R., J.-F.O. and C.R wrote the manuscript. All authors commented on 25 and revised the manuscript.

Competing interests: Authors declare no competing interest.

Materials \& Correspondence: correspondence and material requests should be addressed to C.R and J.-F.O. 


\section{STAR METHODS}

\section{Cell culture.}

Primary mouse embryonic fibroblasts (pMEFs) and primary fetal lung fibroblast (IMR90, ATCC CCL-186) were cultured in Dulbecco's modified Eagle medium (DMEM, Gibco) supplemented with

$510 \%$ of heat-inactivated Fetal Bovine Serum (FBS, Gibco), $100 \mathrm{U} / \mathrm{mL}$ of penicillin and $100 \mu \mathrm{g} / \mathrm{mL}$ of streptomycin (Thermo Fisher Scientific). Cells were routinely passaged $0.05 \%$ Trypsin-EDTA (Thermo Fisher Scientific) and cultured in $20 \% \mathrm{O} 2$ and $8 \% \mathrm{CO} 2$ at $37^{\circ} \mathrm{C}$.

Female EpiSCs (gift from Alice Jouneau) were cultured using chemically defined medium (CDM) as previously defined(Brons et al., 2007), supplemented with Activin A (20ng/mL, Cell Guidance

10 System) and Fgf2 (12 ng/mL, Cell Guidance System). EpiSCs were passaged using $4 \mathrm{mg} / \mathrm{mL}$ Collagenase II (Sigma) and then plated into plates pre-coated with fetal bovine serum.

H9(Thomson et al., 1998) and WIBR2 (Lengner et al., 2010) primed ES cells were cultured on Matrigel-coated culture dishes (BD Biosciences) in mTeSR ${ }^{\mathrm{TM}} 1$ media (Stemcells technologies) according to the manufacturer instructions, in $20 \% \mathrm{O} 2$ and $5 \% \mathrm{CO} 2$ at $37^{\circ} \mathrm{C}$. Primed $\mathrm{hESCs}$ were routinely passaged in clumps using a $0.5 \mathrm{mM}$ EDTA solution as previously described(Beers et al., 2012). For experiments requiring single-cell suspension, cells were incubated with Accutase (Stemcells technologies) and plated in fresh mTeSR ${ }^{\text {TM }} 1$ media supplemented with $10 \mu \mathrm{M}$ of Y -27632 (Stemcells technologies).

Naïve H9 NK2 cells(Takashima et al., 2014) were cultured in 5\% O2 and 5\% CO2 at $37^{\circ} \mathrm{C}$ on $\mathrm{CF} 1$

20 MEFs in a 1:1 mixture of DMEM-F12 and Neurobasal (Thermo Fisher Scientific), 0.5x N2supplement (Thermo Fisher Scientific), 0.5x B27-supplement (Thermo Fisher Scientific), 1x NonEssential Amino Acids (Thermo Fisher Scientific), 2mM L-Glutamine (Thermo Fisher Scientific), 1x Penicillin/Streptomycin (Thermo Fisher Scientific), 0.1mM $\beta$-mercaptoethanol (Sigma-Aldrich), $1 \mu \mathrm{M}$ PD0325901, 1 $\mu \mathrm{M}$ CHIR99021, 20ng/ml human LIF (all from WT-MRC Cambridge Stem Cell Institute) and $2 \mu \mathrm{M}$ Gö6983 (Tocris).

\section{Derivation of primary MEFs.}

pMEFs were derived from 13.5 days post-coitum embryos obtained from crosses of CD1 and Crl:CD1(ICR) mice (Charles River). Embryos were manually cut, further dissociated in 0.05\% Trypsin-EDTA (Thermo Fisher Scientific) and plated on gelatin-coated dishes. At confluence,

pMEFs were frozen until further use (passage 1) and all experiments were performed between passage 1 and 4 , from at least three independent female embryos.

\section{Conversion of primed to naïve hESCs.}

Prior to conversion, primed hESCs were cultured with $\mathrm{mTeSR}^{\mathrm{TM}} 1$ media (see above) in hypoxia ( $5 \%$ $\mathrm{O} 2$ and 5\% CO2) for three passages. Primed hESCs were converted into a naïve-like state using the 
manufacturer. Briefly, resetting was launched using 200000 single-cells plated onto a layer of immortalized MEFs under hypoxic condition. Cells were routinely passaged using TrypLE express enzyme (Thermo Fisher Scientific). RNA samples and cells for RNA-FISH analysis for primed and naïve hESCs were collected at different passages in $\mathrm{mTeSR}^{\mathrm{TM}} 1$ in hypoxia and in expansion medium (day 43/passage 6) respectively. RT-qPCR primers were previously described (Wang et al., 2014).

\section{LNA GapmRs and si-RNAs lipofection.}

All LNA Gapmers (LGs) were designed using the Exiqon online tool (https://www.exiqon.com/) and the siRNA targeting YY1 was previously described(Makhlouf et al., 2014). A non-targeting LG and si-RNA were used as negative controls. LGs were lipofected using the RNAi Max transfection reagent (Invitrogen) according to the manufacturer recommendations. Except for Naïve hESCs, all LNA GapmRs (LGs) experiments were performed at a final concentration of 50nM (30 nM siRNAs) using a reverse transfection protocol. For naïve H9-NK2 hESCs, cells were lipofected twice first at $0 \mathrm{~h}$ by reverse transfection and at $24 \mathrm{~h}$ by forward transfection, with a final concentration of $25 \mathrm{nM}$ of

15 LGs. All samples were collected 48h post-lipofection either in TRIzol for RNA extraction or Laemmli for western blot analysis. The LGs sequences are listed below:

$$
\text { Control AACACGTCTATACGC }
$$

mouse: $\quad$ mLG1 GGACGCCGCCATTTTA

mLG3 CTCTTCTTAATGACAA

$\begin{array}{lll}\text { human: } & \text { hLG1 } & \text { CGTCAGTAGAAGTTAG } \\ & \text { hLG2 } & \text { TCGTCAGTAGAAGTTA } \\ & \text { hLG3 } & \text { TTCGTCAGTAGAAGTT }\end{array}$

\section{Total RNA extraction and RT-qPCR.}

25 Total RNAs were collected using TRIzol (Thermo Fisher Scientific) and extracted following the manufacturer's instruction. RNA Samples were treated using the DNA free Kit (Thermo Fisher Scientific) following the manufacturer recommendations. RNAs were reverse transcribed for $30 \mathrm{~min}$ at $50^{\circ} \mathrm{C}$ using the Superscript IV kit (Thermo Fisher Scientific). cDNAs were diluted 1:5 in water and transcripts expression level was assessed by real-time quantitative PCR (RT-qPCR) using the Power

30 SYBR Green Master Mix (Thermo Fisher Scientific). All samples were run in duplicate on a ViiA-7 real-time thermal cycler (Applied Biosystems). Transcripts RNA levels were normalized against a reference gene following the $2-\Delta \mathrm{Ct}$ method. Unless stated, the Rplp0 gene was used as a reference mouse samples and GAPDH for human samples. All the RT-qPCR primers used in this study are listed in the Table S1. 


\section{Western Blot.}

Total proteins were extracted with Laemmli lysis buffer (4\% SDS; 20\% glycerol; 10\% 2mercaptoethanol; $0.004 \%$ bromophenol blue; $0.125 \mathrm{M}$ Tris- $\mathrm{HCl}$ ) and sonicated on a Bioruptor

5 Sonication System (Diagenode, UCD-200). After $5 \mathrm{~min}$ denaturation at $95^{\circ} \mathrm{C}$, the samples were loaded into a 4-12\% gradient polyacrylamide gel (Invitrogen) for SDS-PAGE electrophoresis and transferred onto Invitrolon PVDF membranes (Invitrogen). The membranes were blocked for $1 \mathrm{~h}$ with 5\% milk in TBST (10 mM Tris, $\mathrm{pH} 8.0,150 \mathrm{mM} \mathrm{NaCl}, 0.5 \%$ Tween 20) and incubated overnight at $4{ }^{\circ} \mathrm{C}$ with antibodies targeting YY1 (1:500, mouse sc-7341, H-10, Santa Cruz Biotechnology) or

10 VINCULIN (1:2000, mouse V9131, Sigma Aldrich) proteins. Proteins of interest were detected using a Peroxidase-conjugated antibody (Goat anti-mouse, 1:10 000, Sigma Aldrich) with the Pierce ECL Western blotting substrate (Thermo Scientific).

\section{Nascent RNA pulldown.}

Nascent RNAs were purified using the Click-iT Nascent RNA capture kit (Invitrogen). Briefly, cells

15 were incubated for $1 \mathrm{~h}$ at $37^{\circ} \mathrm{C}$ with DMEM supplemented with $0.5 \mathrm{mM}$ final of Ethynyl Uridine and total RNAs were extracted using TRIzol reagent. $2 \mu \mathrm{g}$ of total RNAs were used for the biotinylation reaction using $0.5 \mathrm{mM}$ of biotin azide. $1 \mu \mathrm{g}$ of biotinylated RNAs were used for the pulldown assay using Dynabeads MyOne Streptavidin T1 magnetic beads. Reverse transcription was performed using the Superscript VILO cDNA Synthesis Kit (Invitrogen) for $1 \mathrm{~h}$ at $42^{\circ} \mathrm{C}$. cDNAs were diluted 1:2

20 before RT-qPCR to quantify nascent gene expression. For each sample, a condition without EU was processed in parallel (EU-) and a 10\% input (biotinylated RNA before IP) was used to assess enrichment of EU-labelled transcripts after pulldown, in both EU+ and EU- conditions. The values presented in the figures represents $\Delta(-\mathrm{Ct})$ values levels normalized to the nascent level of the $\mathrm{H} 2 \mathrm{~A}$ gene.

\section{RNA-FISH.}

Cells preparation. Naïve H9-NK2 and primed hESCs were grown on coverslips. pMEFs, IMR90 and naïve H9 lines were centrifuged onto SuperfrostPlus slides (VWR) using the Cytospin 3 Cytocentrifuge (Shandon). The cells were fixed for $10 \mathrm{~min}$ in a 3\% Paraformaldehyde solution (Electron Microscopy Science) and permeabilized for 5-10 min in ice-cold CSK buffer (10mM

30 PIPES; 300mM sucrose; $100 \mathrm{mM} \mathrm{NaCl} ; 3 \mathrm{mM} \mathrm{MgCl2;} \mathrm{pH} 6.8$ ) supplemented with $0.5 \%$ Triton X-100 (Sigma-Aldrich), 2mM EGTA (Sigma-Aldrich) and 2mM VRC (New England Biolabs).

Probes preparation. RNA-FISH probes were obtained after Nick translation of fosmids/BAC constructs purified using the Large Construct kit (Qiagen): $1 \mu \mathrm{g}$ of purified DNA was labelled for $3 \mathrm{~h}$ at $15^{\circ} \mathrm{C}$ with fluorescent dUTPs (SpectrumOrange and SpectrumGreen from Abott Molecular and Cy5-UTPs from GE HealthCare Life Science). The templates used in this study are listed below: 
mouse Xist (p510)

mouse $J p x$

human ATRX

human XIST

5 human $J P X$

human $F T X$

Human POLA1

human $X A C T$

mouse Xist intron 1

10 human XIST intron $1 / 2$
Xist genomic locus(Debrand et al., 1998; Rougeulle and Avner, 1996) WI1-1581E8, BACPAC

RP11-42M11, BACPAC

10kb Exon 1 gift from Dr. C. Brown, University of British Columbia. WI2-1796L6, BACPAC

RP11-570B23, BACPAC

RP11-11104L9, BACPAC

RP11-35D3, BACPAC

Oligo-FISH probes were a gift from E. Heard Lab.

Oligo-FISH probes (Stellaris) are listed in Table S1.

Hybridization. $100 \mathrm{ng}$ of probes were supplemented with $1 \mu \mathrm{g}$ of Cot-I DNA (Invitrogen) and/or $3 \mu \mathrm{g}$ of Sheared Salmon Sperm DNA (Invitrogen). After precipitation, the probes were resuspended in deionized formamide (Sigma Aldrich), denatured for $7 \mathrm{~min}$ at $75^{\circ} \mathrm{C}$ and further incubated for $15 \mathrm{~min}$ at $37^{\circ} \mathrm{C}$ if Cot-I DNA was used. Probes were mixed with an equal volume of $2 \mathrm{X}$ Hybridization Buffer

15 (4XSSC, 20\% Dextran Sulfate, 2mg/ml BSA, 2mM VRC). Coverslips were dehydrated in 80-100\% ethanol washes and incubated with the hybridization mix at $37^{\circ} \mathrm{C}$ overnight in a humid chamber. Next, the coverslips were washed for $4 \mathrm{~min}$ at $42^{\circ} \mathrm{C}$ three times with $50 \%$ formaldehyde/2X-SSC (pH7.2) and three times with 2X-SSC. The coverslips were mounted in Vectashield plus DAPI (Vector Laboratories).

\section{Immunofluorescence coupled to RNA-FISH.}

Immunofluorescence coupled to RNA-FISH was performed as described previously(Vallot et al., 2017). The antibodies used for IF are listed below:

primary $\quad \mathrm{H} 3 \mathrm{~K} 27 \mathrm{me} 3$

Upstate, Cat\#07-449

Secondary Alexa Fluor 568nm anti-rabbit antibody Thermo Fisher Scientific, Cat\#A10042

\section{Microscopy and image analysis.}

All fluorescent microscopy images were taken on a fluorescence DMI-6000 inverted microscope with a motorized stage (Leica), equipped with a CCD Camera HQ2 (Roper Scientifics) and a HCX PL APO 100X oil objective (numerical aperture, 1.4, Leica) using the Metamorph software (version 7.04, Roper Scientifics). Depending on the cell line, 30-60 optical z-sections were collected at 0.2, 0.25 or

$300.3 \mu \mathrm{m}$ steps, at different wavelengths depending on the signal (DAPI [360nm, 470nm], FITC [470nm, 525nm], Cy3 [550nm, 570nm], Texas Red [596nm, 612nm] and Cy5 [647nm, 668nm]). Stacks were processed using ImageJ 1.48(Abramoff et al., 2004), and are represented as a 2D "maximum projection" throughout the manuscript. The volume of XIST RNA clouds was assessed on stacks using the plugin 3D object counter from ImageJ(Bolte and Cordelieres, 2006). 
Cellular fractionation was performed on at least 5 millions of cells to allow precise estimation of the cells (V) and nuclei (V') volumes. Fresh pellets of cells were resuspended in 3 volumes (V) of hypotonic buffer (20mM HEPES pH7; 10mM KCl; 0.15mM EDTA; 0.15mM EGTA; 0.15mM spermidine; $0.15 \mathrm{mM}$ spermine). Lysis was performed by adding NP-40 (1\% final, IGEPAL CA-630) and was stopped with the addition of $0.9 \mathrm{~V}$ of SR buffer (50mM HEPES pH 7; 0.25mM EDTA; 10mM $\mathrm{KCl} ; 70 \%$ sucrose; $0.15 \mathrm{mM}$ spermidine; $0.15 \mathrm{mM}$ spermine). The cytosolic fraction was separated from the nuclei by 5 min centrifugation at $4{ }^{\circ} \mathrm{C}, 2000 \mathrm{~g}$ and collected in TRIzol. The pellet of nuclei was washed in $3 \mathrm{~V}$ of nuclei wash buffer (10mM HEPES pH8; 0.1mM EDTA; 100mM NaCl; 25\% glycerol; $0.15 \mathrm{mM}$ spermidine; $0.15 \mathrm{mM}$ spermine) to remove cytoplasmic contaminations. The

10 volume of the nuclei pellet was estimated $\left(\mathrm{V}^{\prime}\right)$ and the nuclei were resuspended in one $\mathrm{V}^{\prime}$ of sucrose buffer (20mM TRIS pH7.65; 60mM NaCl; $15 \mathrm{mM} \mathrm{KCl;0.34M} \mathrm{sucrose;} 0.15 \mathrm{mM}$ spermidine; $0.15 \mathrm{mM}$ spermine). The nuclei were incubated for $30 \mathrm{~min}$ at $4^{\circ} \mathrm{C}$ with $0.29 \mathrm{~V}^{\prime}$ of high salt buffer (900mM NaCl; 20mM TRIS pH7.65; 25\% glycerol; $1.5 \mathrm{mM} \mathrm{MgCl2;0.2mM} \mathrm{EDTA)} \mathrm{to} \mathrm{empty} \mathrm{the}$ nuclei of their soluble content. After 30 min centrifugation at $4{ }^{\circ} \mathrm{C} / 10000 \mathrm{~g}$, the supernatant and the

15 pellet were collected separately in TRIzol, representing respectively the soluble and non-soluble nuclear fractions. After RT-qPCR, the absolute abundance of the transcripts $(\Delta-\mathrm{Ct})$ was normalized to the RNA quantity present in each fraction, from which we computed the abundance of the transcript in a given fraction.

\section{Chromatin immunoprecipitation.}

20 ChIP experiments were performed as described previously(Navarro et al., 2010).

Cells were crosslinked in 1\% Formaldehyde (Cliniscience) for $10 \mathrm{~min}$ and quenched with $0.125 \mathrm{mM}$ glycine for $5 \mathrm{~min}$. Nuclei were extracted after $30 \mathrm{~min}$ incubation in Swelling Buffer (5mM PIPES pH8.0; 85mM KCl; $0.5 \%$ NP-40). Samples were then sonicated in TSE150 buffer $(0.1 \%$ SDS; $1 \%$ Triton; 2mM EDTA; 20mM Tris-HCl pH8; 150mM NaCl) using a Bioruptor Sonication System

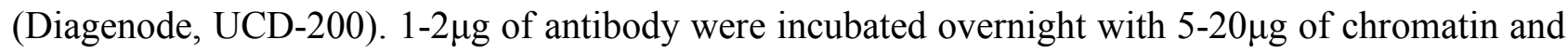
protein A Magnetic Beads (Thermo Scientific). The following mix was then washed in TSE150, TSE500 (20mM Tris-HCl pH8; 2mM EDTA; 0.1\% SDS; 1\% Triton X-100; 500mM NaCl), Washing buffer (10mM Tris-HCl pH8; 1mM EDTA; 250mM LiCl; 0.5\% NP-40; 0.5\% Na-deoxycholate), twice in TE (10mM Tris-HCl pH8; 1mM EDTA) and eluted in TE/1\% SDS. After reverse-crosslink

30 (overnight, $65^{\circ} \mathrm{C}$ ), the samples were purified using a phenol-chloroform extraction, resuspended in water and further analyzed by qPCR in duplicates on both IP and input DNA. All values were processed following the $2-\Delta \mathrm{Ct}$ method and normalized to the input. The primers used for qPCR are available in Table S1. The antibodies used in this study are listed below:

CTCF

35 YY1
Millipore, Cat\#07-729, Lot: 2452497

Abcam, Cat\#ab109237, Lot: GR188694-6 
H3K9me3

Total RNA Pol2

RNA Pol2 CTD Ser5P
Diagenode, Cat\#pAb-193-050, Lot: A1671-001P

Active Motif, Cat\#91151

Active Motif, Cat\#91119

\section{Lentivectors production.}

5 Lentiviral particles were produced by transient transfection of HEK293T cells using the calciumphosphate transfection method. The lentiviral constructs of interest were co-transfected with pMD2.G (Addgene \#12259) and psPAX2 (Addgene \#12260) plasmids (kindly provided by Didier Trono). After $48 \mathrm{~h}$, the culture media was collected, and lentiviral particles were concentrated by ultracentrifugation. For each construct, we assessed the lentiviral titer by infection of HEK293T with serial dilution (1:3) of the lentivirus into DMEM and FACS analysis.

\section{CRISPR inhibition.}

The CRISPR inhibitor system(Gilbert et al., 2013) was used to inhibit $J P X$ transcription in primed H9 hESCs. DNA oligonucleotides corresponding to the sgRNAs sequences were obtained with the online software CCTop (https://crispr.cos.uni-heidelberg.de/index.html). Oligonucleotide pairs were annealed to generate short double-stranded DNA fragments with overhangs compatible with ligation into the BsmbI-digested plasmid pLKO5.sgRNA.EFS.tGFP (Addgene \#57823).

\begin{tabular}{|c|c|}
\hline Sg-A: Ci_hJPX_2_F & CACCGCAATCACTGCGTCCTTACG \\
\hline Ci_hJPX_2_R & AAACCGTAAGGACGCAGTGATTG \\
\hline
\end{tabular}

Sg-B: Ci_hJPX_1_F CACCGACGCCTTGCAACCCCCGTA AAACTACGGGGGTTGCAAGGCGTC

Sg-C: Ci_hJPX_3_F CACCGATCGCGTGGCCTGAGTCGG Ci_hJPX_3_R AAACCCGACTCAGGCCACGCGATC

Production of stable cell lines. H9 cells were infected with the dCas9-mCherry-KRAB construct(Furlan et al., 2018) and sorted by FACS (INFLUX 500-BD BioSciences). A second lentiviral infection was performed with the constructs containing the sgRNAs $(\sim 80-85 \%$ GFP positive).

\section{CRISPR/Cas9-mediated deletion of the $J P X$ promoter region.}

$J P X$ promoter was deleted in primed $\mathrm{H} 9 \mathrm{hESC}$ using the CRISPR-Cas9 system. To proceed, plasmid constructs harboring both the sgRNA sequence and the Cas 9 fused to a reporter gene were used to

30 allow subsequent selection of transfected cells by FACS. sgRNAs downstream of $J P X$ TSS were cloned into a Cas9-GFP construct while upstream guides were cloned into a Cas9-mCherry construct. Therefore, double GFP $+/$ mCherry+ positive cells represent the fraction of cells simultaneously transfected with the two sgRNAs, where the probability for a direct deletion event was increased.

Guides design and cloning. DNA oligonucleotides corresponding to the sgRNAs sequences were obtained with the online software Zifit (http://zifit.partners.org/ZiFiT/ChoiceMenu.aspx). 
Oligonucleotide pairs were annealed to generate short double-stranded DNA fragments with overhangs compatible with the ligation into the BbsI-digested plasmid (pSpCas9(BB)-2A-GFP, Addgene \#48138, Feng Zhang Lab). We also replaced the GFP by a mCherry reporter to produce a pSpCas9(BB)-2A-mCherry plasmid using the NEBuilder HiFi DNA Assembly Cloning Kit (New

5 England Biolabs). The sequences of the guides are listed below:

$\begin{array}{ll}\text { hJU1-F } & \text { CACCGGGGCATGAAGCTTGCACCG } \\ \text { hJU1-R } & \text { AAACCGGTGCAAGCTTCATGCCCC } \\ \text { hJD1-F } & \text { CACCgATGCCATCACTATACATAGT } \\ \text { hJD1-R } & \text { AAACACTATGTATAGTGATGGCATC }\end{array}$

10 hJU3-F CACCGGAAGCATTATTCGAGAAAT

hJU3-R AAACATTTCTCGAATAATGCTTCC

hJD3-F CACCgAAATCTAAGTGATCCATATA

hJD3-R AAACTATATGGATCACTTAGATTTC

Four million of hESCs were transfected with $5 \mu \mathrm{g}$ of plasmid DNA for each guide, using the 4D-

15 Nucleofector system (Lonza) as recommended by the manufacturer. 48h post nucleofection, cells were sorted by FACs (INFLUX 500 BD BioSciences) and double positive GFP+ / mCherry+ cells were plated onto Laminin-521 coated plates (Stemcell technologies) at low density in mTeSR ${ }^{\text {TM}} 1$ supplemented with $1 \mathrm{X}$ CloneR ${ }^{\mathrm{TM}}$ (Stemcell technologies). Individual colonies were picked and screened by PCR for deletions and inversions events using the following primers :

WT allele

hJPXKO_HR-Del F

GGTCCAGGACGTGGAATTTA

hJPXKO_HR-Del_R

TCGTCAATGCAATTTCAAACA

deletion

hJPX-ScrD-Up1

AGGAAAAGTGGGTTTCCACA

hJPX-ScrD-Lo1

GGGTGACAAGAGCAAGACTTC

inversion

hJPX_InvPCR-1F

GGGGAAATGTGAGTGAGTGG

hJPX_InvPCR-1R

GGGGTGCATGTTTAGTTGGT

For each clone, the number of X-chromosomes was validated by qPCR on genomic DNA using:

Outside deletion humanXIC419f ATTTCTACCTTGTACCTAGCACAG humanXIC419r AGATTACATTCAAATCGGAGAGG

Inside deletion humanXIC425f TGTGGGGGTCTCGTAGAAAA humanXIC425r TGCTTCACCGGTAAGGAAAA

\section{Resources for genomic data.}

We downloaded data generated by the ENCODE Project Consortium corresponding to CTCF, RAD21, SMC3, CEPBP, H3K27Ac and H3K4me3 ChIP-seq performed in IMR90 cells; K562 chromatin state hidden Markov model (ChromHMM) and H3K27me3 ChIP-seq and K562. H9 RNAseq (Vallot et al., 2013) and CTCF ChIP-seq (Ji et al., 2016) were obtained from the GEO repository 
under the accession numbers GSM978784, GSE62562 and GSE69646 respectively. We obtained sequence conservation of the human XIC from the UCSC Genome Browser (Kent et al., 2002) corresponding to the 100 vertebrates Base-wise Conservation by PhyloP.

All heatmap from in situ Hi-C datasets (Bonev et al., 2017; Dekker et al., 2017; Rao et al., 2014) represents raw observed matrix visualized at a $5 \mathrm{~kb}$ resolution and were visualized using the Juicebox suit (Durand et al., 2016a). Domains and loops coordinates were obtained from the corresponding studies.

For ChiA-PET datasets, long-range chromatin interactions and signals tracks were obtained from: (i) the ENCODE project (https://www.encodeproject.org) for POLR2A (ENCSR000BZY) and CTCF

10 (ENCSR000CAC) (Li et al., 2012); (ii) the GEO repository for SMC1 ChIA-PET (GSE69643) (Ji et al., 2016). SMC1 ChIA-PET was processed using Juicer Tools (Durand et al., 2016b) for heatmap visualization. All datas were visualized with the Integrative Genomics Viewer (Robinson et al., 2011) or the UCSC Genome browser(Kent et al., 2002) or on the WashU Epigenome Browser (Zhou et al., 2013; Zhou et al., 2011)).

\section{Resources for single-cell RNA-Seq.}

RPKM (Reads Per Kilobase Millions) tables of single-cell RNAseq datasets performed on human embryos (Petropoulos et al., 2016) were obtained from a previous analysis (Vallot et al., 2017). Briefly, RPKM values were computed following a gene-based model and counts falling on regions overlapping two genes were discarded. Unless stated, we used $\log 2(\mathrm{RPKM}+0.001)$ as expression

20 levels for representation and for computation of Pearson's correlation scores. For lineage assignments, we used the metadata from (Stirparo et al., 2018). All graphical plots were obtained using R (version 3.0.2) with the ggplot2 package (version 1.0.1).

\section{Statistical information.}

Throughout the manuscript, RT-qPCR barplots are presented as the mean value with error bars

25 corresponding to standard deviation. The exact number of biological replicates are indicated by the value "n". Statistical tests used to compute statistical significance are specified in figures legend. 


\section{REFERENCES}

Abramoff, M.D., Magalhaes, P.J., and Ram, S.J. (2004). Image Processing with ImageJ. Biophotonics International 11, 36-42.

Beane, R.L., Ram, R., Gabillet, S., Arar, K., Monia, B.P., and Corey, D.R. (2007). Inhibiting gene expression with locked nucleic acids (LNAs) that target chromosomal DNA. Biochemistry 46, 75727580.

Beers, J., Gulbranson, D.R., George, N., Siniscalchi, L.I., Jones, J., Thomson, J.A., and Chen, G. (2012). Passaging and colony expansion of human pluripotent stem cells by enzyme-free dissociation in chemically defined culture conditions. Nat Protoc 7, 2029-2040.

Bolte, S., and Cordelieres, F.P. (2006). A guided tour into subcellular colocalization analysis in light microscopy. J Microsc 224, 213-232.

Bonev, B., Mendelson Cohen, N., Szabo, Q., Fritsch, L., Papadopoulos, G.L., Lubling, Y., Xu, X., Lv, X., Hugnot, J.P., Tanay, A., et al. (2017). Multiscale 3D Genome Rewiring during Mouse Neural Development. Cell 171, 557-572 e524.

Brons, I.G., Smithers, L.E., Trotter, M.W., Rugg-Gunn, P., Sun, B., Chuva de Sousa Lopes, S.M., Howlett, S.K., Clarkson, A., Ahrlund-Richter, L., Pedersen, R.A., et al. (2007). Derivation of pluripotent epiblast stem cells from mammalian embryos. Nature 448, 191-195.

Carmona, S., Lin, B., Chou, T., Arroyo, K., and Sun, S. (2018). LncRNA Jpx induces Xist expression in mice using both trans and cis mechanisms. PLoS Genet 14, e1007378.

Carrel, L., Cottle, A.A., Goglin, K.C., and Willard, H.F. (1999). A first-generation X-inactivation profile of the human X chromosome. Proc Natl Acad Sci 96, 14440-14444.

Cho, S.W., Xu, J., Sun, R., Mumbach, M.R., Carter, A.C., Chen, Y.G., Yost, K.E., Kim, J., He, J., Nevins, S.A., et al. (2018). Promoter of lncRNA Gene PVT1 Is a Tumor-Suppressor DNA Boundary Element. Cell 173, 1398-1412 e1322.

Chureau, C., Prissette, M., Bourdet, A., Barbe, V., Cattolico, L., Jones, L., Eggen, A., Avner, P., and Duret, L. (2002). Comparative sequence analysis of the X-inactivation center region in mouse, human, and bovine. Genome Res 12, 894-908.

Debrand, E., Heard, E., and Avner, P. (1998). Cloning and localization of the murine Xpct gene:

evidence for complex rearrangements during the evolution of the region around the Xist gene. Genomics 48, 296-303.

Dekker, J., Belmont, A.S., Guttman, M., Leshyk, V.O., Lis, J.T., Lomvardas, S., Mirny, L.A., O'Shea, C.C., Park, P.J., Ren, B., et al. (2017). The 4D nucleome project. Nature 549, 219-226.

Dowen, J.M., Fan, Z.P., Hnisz, D., Ren, G., Abraham, B.J., Zhang, L.N., Weintraub, A.S., Schujiers, J., Lee, T.I., Zhao, K., et al. (2014). Control of cell identity genes occurs in insulated neighborhoods in mammalian chromosomes. Cell 159, 374-387.

Durand, N.C., Robinson, J.T., Shamim, M.S., Machol, I., Mesirov, J.P., Lander, E.S., and Aiden, E.L. (2016a). Juicebox Provides a Visualization System for Hi-C Contact Maps with Unlimited Zoom. Cell Syst 3, 99-101.

40 Durand, N.C., Shamim, M.S., Machol, I., Rao, S.S., Huntley, M.H., Lander, E.S., and Aiden, E.L. (2016b). Juicer Provides a One-Click System for Analyzing Loop-Resolution Hi-C Experiments. Cell Syst 3, 95-98.

Duret, L., Chureau, C., Samain, S., Weissenbach, J., and Avner, P. (2006). The Xist RNA gene evolved in eutherians by pseudogenization of a protein-coding gene. Science 312, 1653-1655.

45 Elisaphenko, E.A., Kolesnikov, N.N., Shevchenko, A.I., Rogozin, I.B., Nesterova, T.B., Brockdorff, N., and Zakian, S.M. (2008). A dual origin of the Xist gene from a protein-coding gene and a set of transposable elements. PLoS One 3, e2521.

Engreitz, J.M., Haines, J.E., Perez, E.M., Munson, G., Chen, J., Kane, M., McDonel, P.E., Guttman, M., and Lander, E.S. (2016). Local regulation of gene expression by lncRNA promoters, transcription and splicing. Nature 539, 452-455. 
Furlan, G., Gutierrez Hernandez, N., Huret, C., Galupa, R., van Bemmel, J.G., Romito, A., Heard, E., Morey, C., and Rougeulle, C. (2018). The Ftx Noncoding Locus Controls X Chromosome Inactivation Independently of Its RNA Products. Mol Cell 70, 462-472 e468.

Furlan, G., and Rougeulle, C. (2016). Function and evolution of the long noncoding RNA circuitry orchestrating X-chromosome inactivation in mammals. Wiley Interdiscip Rev RNA 7, 702-722.

Gilbert, L.A., Larson, M.H., Morsut, L., Liu, Z., Brar, G.A., Torres, S.E., Stern-Ginossar, N., Brandman, O., Whitehead, E.H., Doudna, J.A., et al. (2013). CRISPR-mediated modular RNAguided regulation of transcription in eukaryotes. Cell 154, 442-451.

Giorgetti, L., Galupa, R., Nora, E.P., Piolot, T., Lam, F., Dekker, J., Tiana, G., and Heard, E. (2014).

Predictive polymer modeling reveals coupled fluctuations in chromosome conformation and transcription. Cell 157, 950-963.

Guo, G., von Meyenn, F., Rostovskaya, M., Clarke, J., Dietmann, S., Baker, D., Sahakyan, A., Myers, S., Bertone, P., Reik, W., et al. (2017). Epigenetic resetting of human pluripotency. Development 144, 2748-2763.

15 Hezroni, H., Ben-Tov Perry, R., Meir, Z., Housman, G., Lubelsky, Y., and Ulitsky, I. (2017). A subset of conserved mammalian long non-coding RNAs are fossils of ancestral protein-coding genes. Genome Biol 18, 162.

Hezroni, H., Koppstein, D., Schwartz, M.G., Avrutin, A., Bartel, D.P., and Ulitsky, I. (2015). Principles of long noncoding RNA evolution derived from direct comparison of transcriptomes in 17 species. Cell Rep 11, 1110-1122.

Hnisz, D., Day, D.S., and Young, R.A. (2016). Insulated Neighborhoods: Structural and Functional Units of Mammalian Gene Control. Cell 167, 1188-1200.

Ji, X., Dadon, D.B., Powell, B.E., Fan, Z.P., Borges-Rivera, D., Shachar, S., Weintraub, A.S., Hnisz, D., Pegoraro, G., Lee, T.I., et al. (2016). 3D Chromosome Regulatory Landscape of Human 25 Pluripotent Cells. Cell Stem Cell 18, 262-275.

Johnston, C.M., Newall, A.E., Brockdorff, N., and Nesterova, T.B. (2002). Enox, a novel gene that maps $10 \mathrm{~kb}$ upstream of Xist and partially escapes X inactivation. Genomics 80, 236-244.

Karner, H.M., Webb, C., Carmona, S., Liu, Y., Lin, B., Erhard, M., Chan, D., Baldi, P., Spitale, R.C., and Sun, S. (2019). Functional conservation of lncRNA JPX despite sequence and structural 30 divergence. bioRxiv 686113.

Kent, W.J., Sugnet, C.W., Furey, T.S., Roskin, K.M., Pringle, T.H., Zahler, A.M., and Haussler, D. (2002). The human genome browser at UCSC. Genome Res 12, 996-1006.

Kolesnikov, N.N., and Elisaphenko, E.A. (2010). Comparative organization and the origin of noncoding regulatory RNA genes from X-chromosome inactivation center of human and mouse.

35 Russian Journal of Genetics 46, 1223-1228.

Lengner, C.J., Gimelbrant, A.A., Erwin, J.A., Cheng, A.W., Guenther, M.G., Welstead, G.G., Alagappan, R., Frampton, G.M., Xu, P., Muffat, J., et al. (2010). Derivation of pre-X inactivation human embryonic stem cells under physiological oxygen concentrations. Cell 141, 872-883.

Leucci, E., Vendramin, R., Spinazzi, M., Laurette, P., Fiers, M., Wouters, J., Radaelli, E., Eyckerman,

40 S., Leonelli, C., Vanderheyden, K., et al. (2016). Melanoma addiction to the long non-coding RNA SAMMSON. Nature 531, 518-522.

Li, G., Ruan, X., Auerbach, R.K., Sandhu, K.S., Zheng, M., Wang, P., Poh, H.M., Goh, Y., Lim, J., Zhang, J., et al. (2012). Extensive promoter-centered chromatin interactions provide a topological basis for transcription regulation. Cell 148, 84-98.

45 Lin, N., Chang, K.Y., Li, Z., Gates, K., Rana, Z.A., Dang, J., Zhang, D., Han, T., Yang, C.S., Cunningham, T.J., et al. (2014). An evolutionarily conserved long noncoding RNA TUNA controls pluripotency and neural lineage commitment. Mol Cell 53, 1005-1019.

Luo, S., Lu, J.Y., Liu, L., Yin, Y., Chen, C., Han, X., Wu, B., Xu, R., Liu, W., Yan, P., et al. (2016). Divergent lncRNAs Regulate Gene Expression and Lineage Differentiation in Pluripotent Cells. Cell Stem Cell 18,637-652. 
Makhlouf, M., Ouimette, J.F., Oldfield, A., Navarro, P., Neuillet, D., and Rougeulle, C. (2014). A prominent and conserved role for YY1 in Xist transcriptional activation. Nature communications 5, 4878.

Mekhoubad, S., Bock, C., de Boer, A.S., Kiskinis, E., Meissner, A., and Eggan, K. (2012). Erosion of dosage compensation impacts human iPSC disease modeling. Cell Stem Cell 10, 595-609.

Navarro, P., Oldfield, A., Legoupi, J., Festuccia, N., Dubois, A., Attia, M., Schoorlemmer, J., Rougeulle, C., Chambers, I., and Avner, P. (2010). Molecular coupling of Tsix regulation and pluripotency. Nature 468, 457-460.

Navarro, P., Pichard, S., Ciaudo, C., Avner, P., and Rougeulle, C. (2005). Tsix transcription across the Xist gene alters chromatin conformation without affecting Xist transcription: implications for Xchromosome inactivation. Genes Dev 19, 1474-1484.

Necsulea, A., Soumillon, M., Warnefors, M., Liechti, A., Daish, T., Zeller, U., Baker, J.C., Grutzner, F., and Kaessmann, H. (2014). The evolution of lncRNA repertoires and expression patterns in tetrapods. Nature 505, 635-640.

15 Nora, E.P., Lajoie, B.R., Schulz, E.G., Giorgetti, L., Okamoto, I., Servant, N., Piolot, T., van Berkum, N.L., Meisig, J., Sedat, J., et al. (2012). Spatial partitioning of the regulatory landscape of the Xinactivation centre. Nature 485, 381-385.

Okamoto, I., Patrat, C., Thepot, D., Peynot, N., Fauque, P., Daniel, N., Diabangouaya, P., Wolf, J.P., Renard, J.P., Duranthon, V., et al. (2011). Eutherian mammals use diverse strategies to initiate Xchromosome inactivation during development. Nature 472, 370-374.

Paralkar, V.R., Taborda, C.C., Huang, P., Yao, Y., Kossenkov, A.V., Prasad, R., Luan, J., Davies, J.O., Hughes, J.R., Hardison, R.C., et al. (2016). Unlinking an lncRNA from Its Associated cis Element. Mol Cell 62, 104-110.

Petropoulos, S., Edsgard, D., Reinius, B., Deng, Q., Panula, S.P., Codeluppi, S., Reyes, A.P., Linnarsson, S., Sandberg, R., and Lanner, F. (2016). Single-Cell RNA-Seq Reveals Lineage and X Chromosome Dynamics in Human Preimplantation Embryos. Cell 167, 285.

Rao, S.S., Huntley, M.H., Durand, N.C., Stamenova, E.K., Bochkov, I.D., Robinson, J.T., Sanborn, A.L., Machol, I., Omer, A.D., Lander, E.S., et al. (2014). A 3D map of the human genome at kilobase resolution reveals principles of chromatin looping. Cell 159, 1665-1680.

30 Romito, A., and Rougeulle, C. (2011). Origin and evolution of the long non-coding genes in the Xinactivation center. Biochimie 93, 1935-1942.

Rougeulle, C., and Avner, P. (1996). Cloning and characterization of a murine brain specific gene $B p x$ and its human homologue lying within the Xic candidate region. Hum Mol Genet 5, 41-49.

Sahakyan, A., Kim, R., Chronis, C., Sabri, S., Bonora, G., Theunissen, T.W., Kuoy, E., Langerman, J., Clark, A.T., Jaenisch, R., et al. (2017). Human Naive Pluripotent Stem Cells Model X Chromosome Dampening and X Inactivation. Cell Stem Cell 20, 87-101.

Shin, J., Bossenz, M., Chung, Y., Ma, H., Byron, M., Taniguchi-Ishigaki, N., Zhu, X., Jiao, B., Hall, L.L., Green, M.R., et al. (2010). Maternal Rnf12/RLIM is required for imprinted X-chromosome inactivation in mice. Nature 467, 977-981.

40 Stirparo, G.G., Boroviak, T., Guo, G., Nichols, J., Smith, A., and Bertone, P. (2018). Integrated analysis of single-cell embryo data yields a unified transcriptome signature for the human preimplantation epiblast. Development 145.

Stojic, L., Lun, A.T.L., Mangei, J., Mascalchi, P., Quarantotti, V., Barr, A.R., Bakal, C., Marioni, J.C., Gergely, F., and Odom, D.T. (2018). Specificity of RNAi, LNA and CRISPRi as loss-offunction methods in transcriptional analysis. Nucleic Acids Res 46, 5950-5966.

Sun, F., Chronis, C., Kronenberg, M., Chen, X.F., Su, T., Lay, F.D., Plath, K., Kurdistani, S.K., and Carey, M.F. (2019). Promoter-Enhancer Communication Occurs Primarily within Insulated Neighborhoods. Mol Cell 73, 250-263 e255.

Sun, S., Del Rosario, B.C., Szanto, A., Ogawa, Y., Jeon, Y., and Lee, J.T. (2013). Jpx RNA activates Xist by evicting CTCF. Cell 153, 1537-1551. 
Takashima, Y., Guo, G., Loos, R., Nichols, J., Ficz, G., Krueger, F., Oxley, D., Santos, F., Clarke, J., Mansfield, W., et al. (2014). Resetting transcription factor control circuitry toward ground-state pluripotency in human. Cell 158, 1254-1269.

Tesar, P.J., Chenoweth, J.G., Brook, F.A., Davies, T.J., Evans, E.P., Mack, D.L., Gardner, R.L., and McKay, R.D. (2007). New cell lines from mouse epiblast share defining features with human embryonic stem cells. Nature 448, 196-199.

Theunissen, T.W., Friedli, M., He, Y., Planet, E., O'Neil, R.C., Markoulaki, S., Pontis, J., Wang, H., Iouranova, A., Imbeault, M., et al. (2016). Molecular Criteria for Defining the Naive Human Pluripotent State. Cell Stem Cell 19, 502-515.

10 Thomson, J.A., Itskovitz-Eldor, J., Shapiro, S.S., Waknitz, M.A., Swiergiel, J.J., Marshall, V.S., and Jones, J.M. (1998). Embryonic stem cell lines derived from human blastocysts. Science 282, 11451147.

Tian, D., Sun, S., and Lee, J.T. (2010). The long noncoding RNA, Jpx, is a molecular switch for X chromosome inactivation. Cell 143, 390-403.

15 Tripathi, V., Ellis, J.D., Shen, Z., Song, D.Y., Pan, Q., Watt, A.T., Freier, S.M., Bennett, C.F., Sharma, A., Bubulya, P.A., et al. (2010). The nuclear-retained noncoding RNA MALAT1 regulates alternative splicing by modulating SR splicing factor phosphorylation. Mol Cell 39, 925-938.

Ulitsky, I., Shkumatava, A., Jan, C.H., Sive, H., and Bartel, D.P. (2011). Conserved function of lincRNAs in vertebrate embryonic development despite rapid sequence evolution. Cell 147, 1537 1550.

Vallot, C., Huret, C., Lesecque, Y., Resh, A., Oudrhiri, N., Bennaceur-Griscelli, A., Duret, L., and Rougeulle, C. (2013). XACT, a long non-coding transcript coating the active $\mathrm{X}$ in human pluripotent cells. Nat Genet 45, 239-241.

Vallot, C., Ouimette, J.F., Makhlouf, M., Feraud, O., Pontis, J., Come, J., Martinat, C., BennaceurGriscelli, A., Lalande, M., and Rougeulle, C. (2015). Erosion of X Chromosome Inactivation in Human Pluripotent Cells Initiates with XACT Coating and Depends on a Specific Heterochromatin Landscape. Cell Stem Cell 16, 533-546.

Vallot, C., Ouimette, J.F., and Rougeulle, C. (2016). Establishment of X chromosome inactivation and epigenomic features of the inactive $\mathrm{X}$ depend on cellular contexts. Bioessays 38, 869-880.

Vallot, C., Patrat, C., Collier, A.J., Huret, C., Casanova, M., Liyakat Ali, T.M., Tosolini, M., Frydman, N., Heard, E., Rugg-Gunn, P.J., et al. (2017). XACT Noncoding RNA Competes with XIST in the Control of X Chromosome Activity during Human Early Development. Cell Stem Cell 20, 102111.

Villar, D., Berthelot, C., Aldridge, S., Rayner, T.F., Lukk, M., Pignatelli, M., Park, T.J., Deaville, R., Erichsen, J.T., Jasinska, A.J., et al. (2015). Enhancer evolution across 20 mammalian species. Cell 160, 554-566.

Wang, J., Xie, G., Singh, M., Ghanbarian, A.T., Rasko, T., Szvetnik, A., Cai, H., Besser, D., Prigione, A., Fuchs, N.V., et al. (2014). Primate-specific endogenous retrovirus-driven transcription defines naive-like stem cells. Nature 516, 405-409.

40 Washietl, S., Kellis, M., and Garber, M. (2014). Evolutionary dynamics and tissue specificity of human long noncoding RNAs in six mammals. Genome Res 24, 616-628.

Yan, L., Yang, M., Guo, H., Yang, L., Wu, J., Li, R., Liu, P., Lian, Y., Zheng, X., Yan, J., et al. (2013). Single-cell RNA-Seq profiling of human preimplantation embryos and embryonic stem cells. Nat Struct Mol Biol 20, 1131-1139.

45 Zhou, X., Lowdon, R.F., Li, D., Lawson, H.A., Madden, P.A., Costello, J.F., and Wang, T. (2013). Exploring long-range genome interactions using the WashU Epigenome Browser. Nat Methods 10, 375-376.

Zhou, X., Maricque, B., Xie, M., Li, D., Sundaram, V., Martin, E.A., Koebbe, B.C., Nielsen, C., Hirst, M., Farnham, P., et al. (2011). The Human Epigenome Browser at Washington University. Nat Methods 8, 989-990. 